\title{
Fouilles de Pachi (Mégare)
}

Rapport de la campagne 2021

Thierry Lucas, Patrick Marchetti, Panagiota Avgerinou, Lou de Barbarin, Jules Buffet, Anthony Peeters, Louis Pirat, Sylvain Grosfilley, Brieuc Guillaume et Marilou de Vals

\section{OpenEdition}

12 Journals

Édition électronique

URL : https://journals.openedition.org/baefe/4610

DOI : $10.4000 /$ baefe. 4610

ISSN : 2732-687X

Éditeur

ResEFE

\section{Référence électronique}

Thierry Lucas, Patrick Marchetti, Panagiota Avgerinou, Lou de Barbarin, Jules Buffet, Anthony Peeters, Louis Pirat, Sylvain Grosfilley, Brieuc Guillaume et Marilou de Vals, «Fouilles de Pachi (Mégare)» [notice archéologique], Bulletin archéologique des Écoles françaises à l'étranger [En ligne], Grèce, mis en ligne le 30 novembre 2021, consulté le 01 décembre 2021. URL : http://journals.openedition.org/ baefe/4610; DOI : https://doi.org/10.4000/baefe.4610

Ce document a été généré automatiquement le 1 décembre 2021

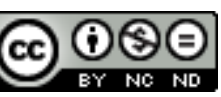

Le Bulletin archéologique des Écoles françaises à l'étranger est mise à disposition selon les termes de la Licence Creative Commons Attribution - Pas d'Utilisation Commerciale - Pas de Modification 4.0 International. 


\section{Fouilles de Pachi (Mégare)}

Rapport de la campagne 2021

Thierry Lucas, Patrick Marchetti, Panagiota Avgerinou, Lou de Barbarin, Jules Buffet, Anthony Peeters, Louis Pirat, Sylvain Grosfilley, Brieuc Guillaume et Marilou de Vals

\section{NOTE DE L'AUTEUR}

Date précise de l'opération : 23 août - 17 septembre 2021

Numéro de mission : I40

Composition de l'équipe de terrain : Isabella Bergamini (Università di Bologna), fouilleuse; Jules Buffet (Université Paris-Nanterre), responsable de secteur ; Lou de Barbarin (Aix-Marseille Université), céramologue, responsable d'apothèque ; Marilou de Vals (Sorbonne Université), co-responsable d'apothèque ; Alexis Dhenain (Sorbonne Université), fouilleur ; Sylvain Grosfilley, archéologue ; Alexis Groussaud (Université Paris 1 Panthéon-Sorbonne), fouilleur ; Brieuc Guillaume (EFA), topographe ; PierreIdriss Lagourgue (École Normale Supérieure Paris-Saclay), fouilleur ; Anaïs Marchand (Université Paris 1 Panthéon-Sorbonne), fouilleuse ; Despina Moschou (Éphorie des antiquités d'Attique occidentale), archéologue ; Anthony Peeters (Université Catholique de Louvain), responsable de secteur ; Louis Pirat (INRAP), responsable de secteur ; Axel Neau (Université Montpellier 3), fouilleur ; Kostas Svigkos (Éphorie des antiquités d'Attique occidentale), ouvrier ; Panagiotis Tsilivigkos (Éphorie des antiquités d'Attique occidentale), ouvrier ; Sarah Vyverman (Université Catholique de Louvain), fouilleuse.

Établissement éditeur : EFA

Établissements porteurs de l'opération : EFA, Éphorie des Antiquités d'Attique occidentale

Chroniques de l'EFA :

Mégare, Pachis 2017

Mégare, Pachis, Aghios Georgios, 2018 


\section{Les fouilles de Pachi en 2021 : objectifs et moyens}

\section{Historique des recherches : les campagnes de 2017 et 2018}

1 Les opérations de terrain à Pachi ont pris la forme, dès 2017, d'une collaboration entre l'École française d'Athènes et l'Éphorie des Antiquités d'Attique occidentale. L'enjeu était de mener une exploration archéologique complète, incluant prospections et fouilles, des vestiges antiques de Pachi, et en particulier des fortifications visibles sur la colline d'Agios Georgios et de la colline de Palaiokastro. Entre ces deux collines se situait dans l'Antiquité, en toute probabilité, le port de Nisaia, connecté à Mégare par des Longs-Murs. L'enjeu des opérations était donc de préciser la topographie et la chronologie de l'occupation antique dans ce secteur encore mal connu de la cité antique de Mégare.

2 Deux campagnes de terrain ont eu lieu, en 2017 et 2018, sous la direction conjointe de François-Dominique Deltenre (membre belge de l'EFA) et Panagiota Avgerinou (Musée de Mégare). Ces deux campagnes ont permis de mener à bien la prospection complète de la colline d'Agios Georgios, où quatre sondages ont en outre été pratiqués (fig. 1). Même si l'étude définitive du matériel n'a pu être menée à bien, les données préliminaires indiquent une fondation des fortifications sur la colline au $\mathrm{v}^{\mathrm{e}} \mathrm{ou}$ au IV siècle av.J.-C. La prospection confirme une importante occupation classique et hellénistique, mais elle a également permis de relever du matériel plus ancien, jusqu'au $\mathrm{VIII}^{\mathrm{e}}$ siècle av. J.-C. Le site est réoccupé à l'époque proto-byzantine ; le matériel d'époque romaine, en revanche, fait défaut, marquant un hiatus dans l'occupation du site à cette époque. Lors de la campagne 2018, trois sondages ont également été entamés sur la colline de Palaiokastro (PAL-1, PAL-2, PAL-3) ; tous trois étaient situés au pied du mur du kastro (fig. 2, en rouge). Aucun de ces sondages n'avait pu être terminé en raison des intempéries, et les opérations avaient essentiellement permis de dégager d'épaisses couches de démolition et d'atteindre en PAL-1 un niveau plus homogène, vraisemblablement un niveau de circulation contemporain du dernier état du mur ${ }^{1}$. Une ultime campagne prévue en 2019 n'ayant pu avoir lieu, le dossier est ensuite resté en sommeil jusqu'en 2021. 
Fig. 1. Plan général de la colline d'Agios Georgios montrant l'implantation de la grille de prospection et des sondages de 2017-2018.

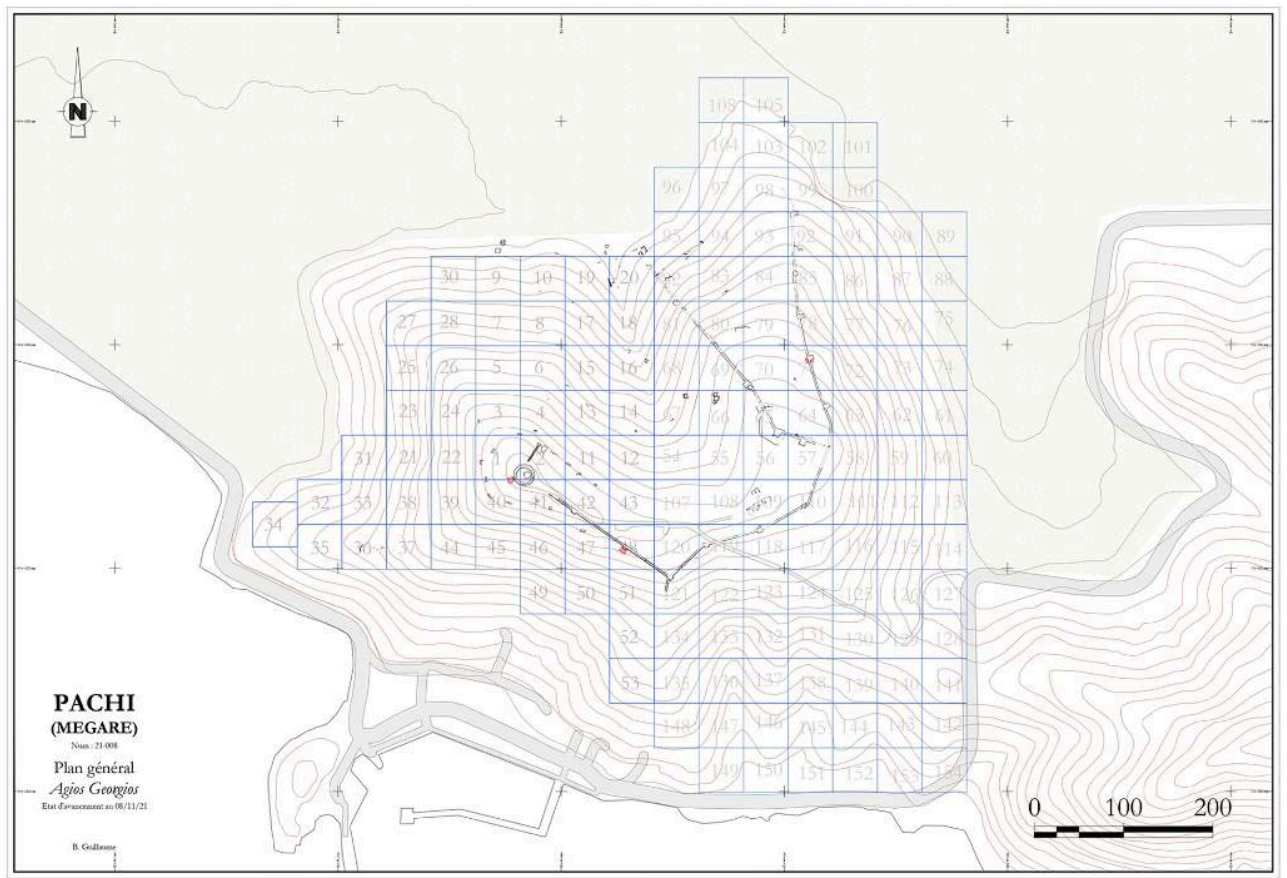

Topographie et DAO : B. Guillaume.

Fig. 2. Plan général de la colline de Palaiokastro montrant l'implantation des sondages de 2018 (en rouge) et 2021 (en vert).



Topographie et DAO : B. Guillaume. 


\section{La campagne de 2021}

3 La reprise des fouilles de 2021 a vu un changement dans l'encadrement de l'équipe : la campagne a de nouveau pris la forme d'une collaboration avec les services de l'éphorie, toujours représentés par Panagiota Avgerinou, tandis que la direction française de la mission était confiée à Patrick Marchetti et à Thierry Lucas, membre scientifique de deuxième année, qui a assuré l'essentiel de la préparation de la mission et de sa mise en œuvre sur le terrain.

4 L'objectif de la campagne de 2021 était simple : il s'agissait surtout de clôturer les travaux entrepris en 2018 à Palaiokastro, en reprenant pour l'essentiel la trame de la campagne prévue en 2019. Cela impliquait : 1 . de mener à son terme au moins l'un des sondages de 2018, pour tenter d'établir la chronologie du kastro et voir si celui-ci reposait sur des structures plus anciennes, et 2 . de mener des sondages en contrebas du mur du kastro, pour tester le potentiel archéologique de la colline dans son ensemble; en particulier, il s'agissait de voir si les nombreux blocs antiques remployés dans le mur du kastro provenaient d'une phase de construction antérieure située sur la colline elle-même.

5 La campagne a duré quatre semaines, du 23 août au 17 septembre. Elle a mobilisé, du côté français, une quinzaine de personnes: Thierry Lucas et Patrick Marchetti, responsables de la mission; Jules Buffet, Anthony Peeters et Louis Pirat, responsables de secteur; Brieuc Guillaume, topographe; Lou de Barbarin, céramologue et responsable de l'apothèque; Marilou de Vals, géologue et co-responsable de l'apothèque; un archéologue professionnel, Sylvain Grosfilley; et les étudiants Isabella Bergamini, Alexis Dhenain, Alexis Groussaud, Pierre-Idriss Lagourgue, Anaïs Marchand, Axel Neau et Sarah Vyverman. La partie grecque de la mission, dirigée par Panagiota Avgerinou, a mobilisé une archéologue, Despina Moschou, deux ouvriers, Panagiotis Tsilivigkos et Kostas Svigkos, et une restauratrice, Kyriaki Karpathiotaki.

Concernant le déroulement de la campagne, il a été choisi d'emblée de limiter au strict minimum la reprise des sondages de 2018. En effet, les trois sondages étaient dans une position similaire, au pied du mur du kastro. La reprise intégrale des trois sondages aurait donc nécessité beaucoup d'efforts, pour des résultats probablement identiques d'un sondage à l'autre. Seuls les sondages PAL-1 et PAL-3 ont donc été repris, sur une partie seulement de leur emprise initiale : le sondage PAL-1 par l'équipe française, et le sondage PAL-3 par les ouvriers grecs (ce dernier sondage a été renommé PAL-6; non représenté sur le plan de la fig. 2). Deux secteurs supplémentaires ont été ouverts à la fouille en contrebas de la muraille : PAL-4, en contrebas de l'angle sud-est du kastro, et plus précisément à l'est de ce dernier ; et PAL-5, incluant deux sondages au nord-ouest du kastro (fig. 2, en vert). Ainsi placés, ces deux nouveaux secteurs de fouilles devaient permettre de tester la stratigraphie en deux endroits distincts du kastro, dans des zones où l'on pouvait espérer trouver des niveaux moins perturbés par l'occupation médiévale, ainsi que par l'occupation moderne ; lors de la Seconde Guerre Mondiale, en effet, une batterie d'artillerie anti-aérienne a été installée au sommet du kastro pour protéger les abords du terrain d'aviation voisin, et cette occupation a lourdement affecté le terrain, avec la présence de tranchées pour abriter les défenseurs de la batterie et l'établissement d'abris bétonnés. Les bases en dur des trois pièces d'artillerie de la batterie sont encore visibles au sommet du kastro. 
7 Les secteurs PAL-1, PAL-5 et PAL-6 ont été remblayés en fin de fouille, avec du géotextile et la terre des déblais. Le secteur PAL-4, où l'épaisseur de terre enlevée était généralement très faible et où le substrat a été atteint partout, a été laissé en l'état, ce qui permet en outre de garder un accès aux murs de la tour pour une étude plus approfondie.

\section{Les fouilles au pied du kastro : secteurs PAL-1 (responsable : Anthony Peeters) et PAL-6}

\section{PAL-1 : Situation initiale et implantation du sondage de 2021}

8 Le sondage PAL-1 avait été entrepris en 2018 pour tester l'implantation du kastro. Il se présentait comme un rectangle de $11 \mathrm{~m}$ sur 3 environ, placé entre le mur du kastro à proprement parler (USM PK1) et un mur de moellons parallèle à ce dernier (USM PK2), qu'on peut suivre sur tout le front nord du kastro. En 2018, les opérations avaient permis de dégager une importante couche de démolition constituée de nombreux blocs et fragments architecturaux. Sous cette couche, un niveau de circulation avait été atteint, lequel était coupé dans sa partie occidentale par une tranchée moderne, d'après le matériel trouvé dans son remplissage, étroite et peu profonde. Cette tranchée coupait obliquement le mur USM PK2. Il pourrait s'agir d'une tranchée liée aux opérations de fouilles menées à Palaiokastro dans les années 1930, ou bien d'un aménagement lié à la construction de la batterie d'artillerie. Enfin, dans le mur du kastro à proprement parler, caractérisé dans ce secteur comme partout ailleurs par une abondance de blocs antiques en remploi, une statue avait été découverte; il s'agit de la partie inférieure d'une statue de femme ${ }^{2}$ portant l'himation, de médiocre facture. Elle était placée dans la partie du mur la plus à l'ouest du sondage et avait servi, avec d'autres blocs assez grossiers, à boucher une ouverture du kastro bien identifiable à cet endroit par la présence du départ de deux piédroits et d'un seuil constitué d'un bloc de marbre en remploi. Ce remplissage tranchait de manière assez nette avec le reste du mur, constitué de blocs remployés soigneusement choisis et agencés.

9 L'enjeu principal du sondage en 2021 restait le même qu'en 2018 : atteindre les niveaux les plus bas pour examiner les fondations du kastro et du mur USM PK2 ; et voir si le kastro était établi sur un bâti d'une phase antérieure. Dès le début de la campagne, il a été décidé de ne reprendre le sondage que dans sa partie occidentale, en le réduisant à un carré de 3 mètres sur 3 environ ; la surface ainsi réduite permettait de poursuivre la fouille en profondeur avec des moyens réduits, tout en conservant la possibilité, en fonction des résultats, d'étendre le sondage dans un second temps. La fouille du secteur PAL-1 a occupé trois semaines en tout. Au vu des résultats, il a été décidé de ne pas étendre le sondage.

\section{PAL-1, les opérations de 2021 : déroulement et résultats}

10 Les niveaux fouillés durant la campagne 2021 dans ce secteur peuvent se résumer à deux horizons d'occupation bien distincts: un épais remblai d'époque médiévale ou ottomane, incluant une canalisation d'évacuation des eaux; et dans la partie la plus profonde du sondage, des niveaux helladiques homogènes, au contact avec le substrat rocheux. 

probablement à former une terrasse entre le mur du kastro et le mur USM PK2, qui forme comme un avant-mur parallèle à ce dernier. En l'état, il n'est pas possible de discerner si cet ensemble servait uniquement à la circulation ou s'il avait une fonction défensive.

12

La fouille a permis de mettre au jour, contre le mur du kastro, une canalisation de terre cuite positionnée verticalement, maintenue par une structure faite de moellons et de mortier. De là, l'eau pouvait s'échapper par une ouverture située au bas de cette structure, qui donnait sur un niveau de mortier. Il est difficile de déterminer si l'eau pouvait ensuite être recueillie, ou s'il s'agissait là d'un simple drain - et tout aussi difficile de déterminer si cette structure avait vocation à fonctionner à l'air libre, ou si elle était enterrée.

13 Cette structure, située exactement à l'aplomb de l'endroit où avait été découvert le fragment de statue en 2018, a permis de mieux comprendre la fonction de ce dernier : un trou avait été grossièrement ménagé dans l'espace délimité par les deux pieds de la statue, la plinthe et la bordure inférieure de l'himation; ce trou communiquait avec une conduite de section carrée ménagée dans le blocage du mur du kastro. La statue servait donc d'orifice à une conduite de drainage et permettait aux eaux de s'écouler dans la canalisation située en-dessous (fig. 3). Il est à noter qu'en plusieurs autres endroits, le mur est semblablement percé par des conduites de drainage, signe qu'il s'agissait là d'un élément important dans l'architecture du kastro. À l'aplomb de la conduite découverte en PAL-1, signalons également la présence au sommet du kastro d'une citerne médiévale de grande taille, réaménagée en casemate pendant la Seconde Guerre mondiale.

Fig. 3. Vue générale du sondage PAL-1 en fin de fouilles, avec la structure hydraulique.

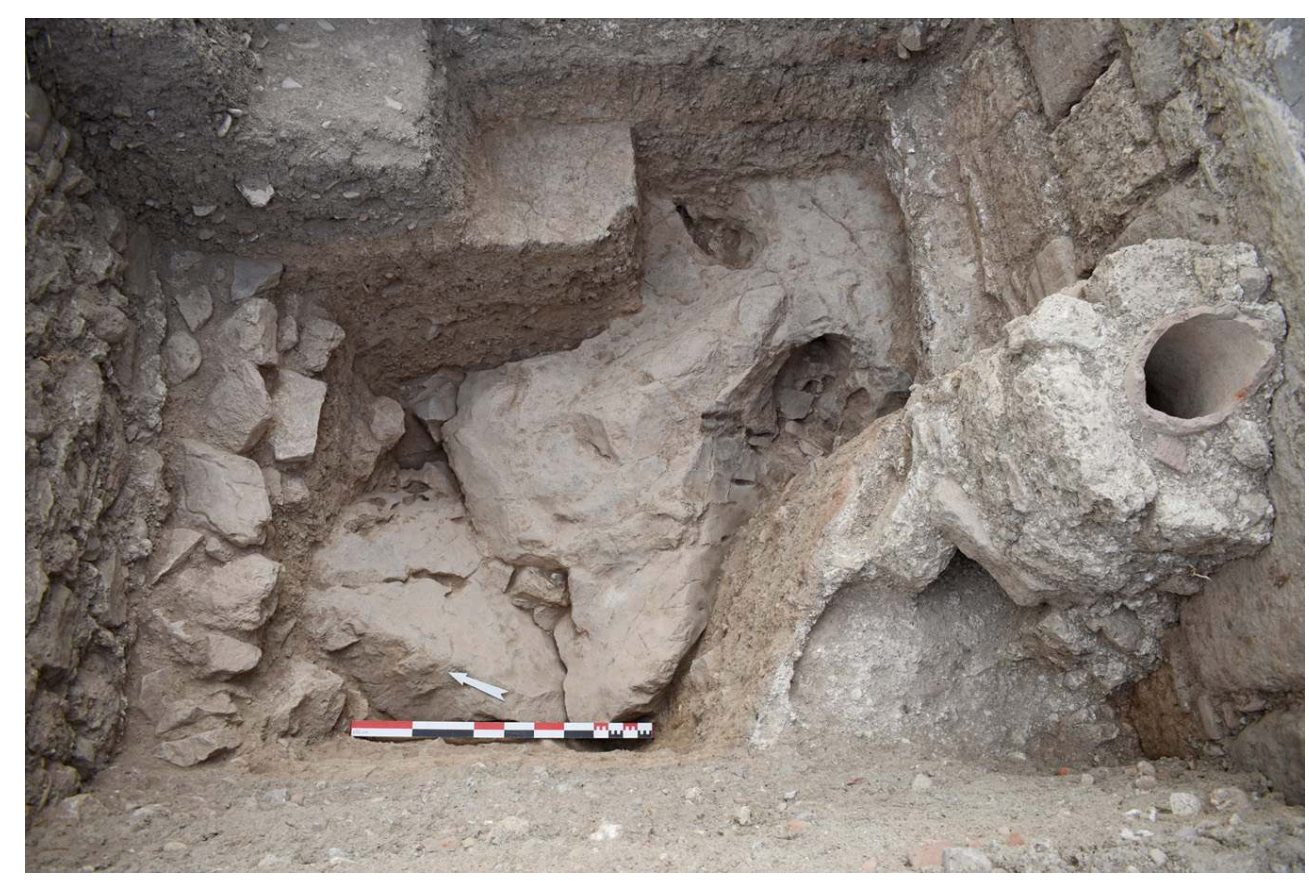

Cliché : A. Peeters.

Bulletin archéologique des Écoles françaises à l'étranger , Grèce 
Le matériel provenant de la fouille de ce remblai est très hétérogène ; l'ensemble peut cependant être daté de façon certaine de la période médiévale ou ottomane, en raison de la présence de céramique glaçurée jusqu'au niveau d'assise de la structure hydraulique ; certains éléments sont indéniablement ottomans. Les couches concernées ont également livré des quantités importantes de céramique plus ancienne, qui doit être interprétée comme le résidu de phases antérieures. Selon toute vraisemblance, cette céramique provient des terres utilisées pour le remblai (fig. 4 et 5 ).

Fig. 4. Sélection de céramique préhistorique et antique de l'US 1012.

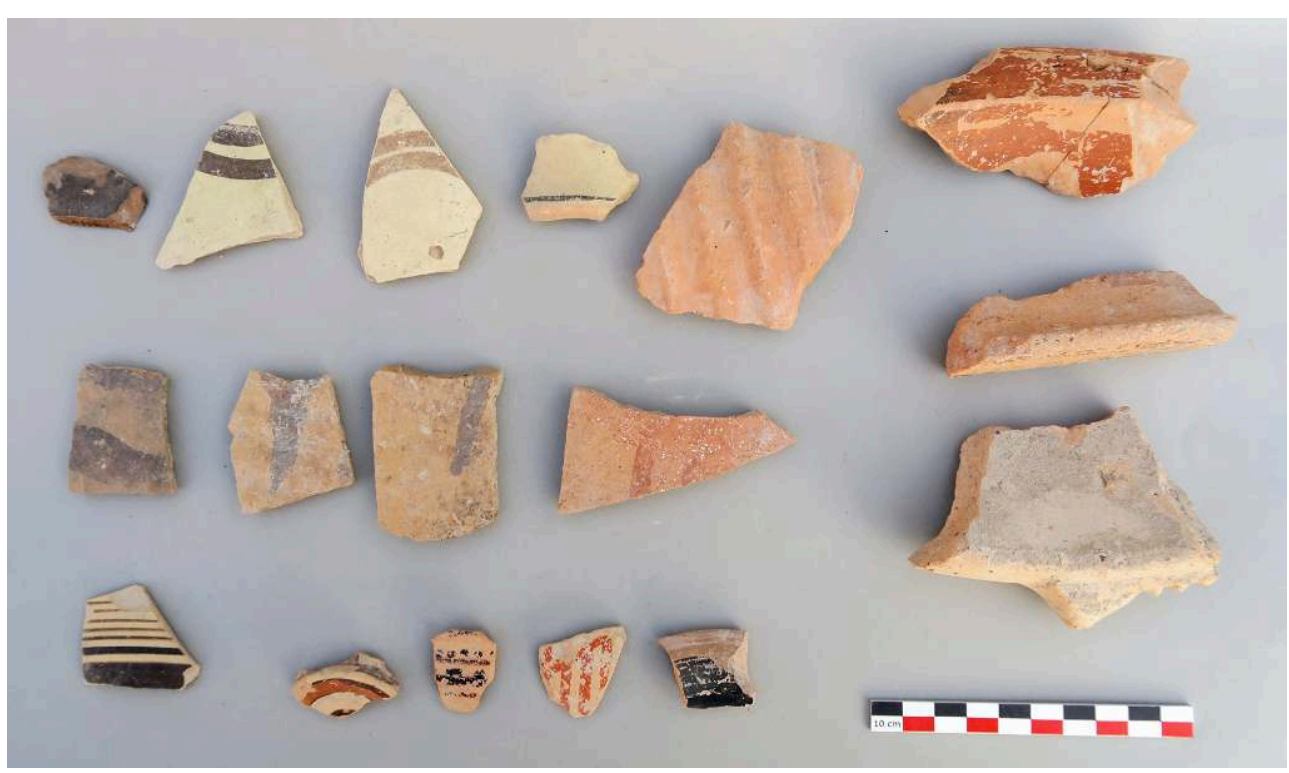

Cliché : L. de Barbarin. 
Fig. 5. Sélection de céramique byzantine de l'US 1012.

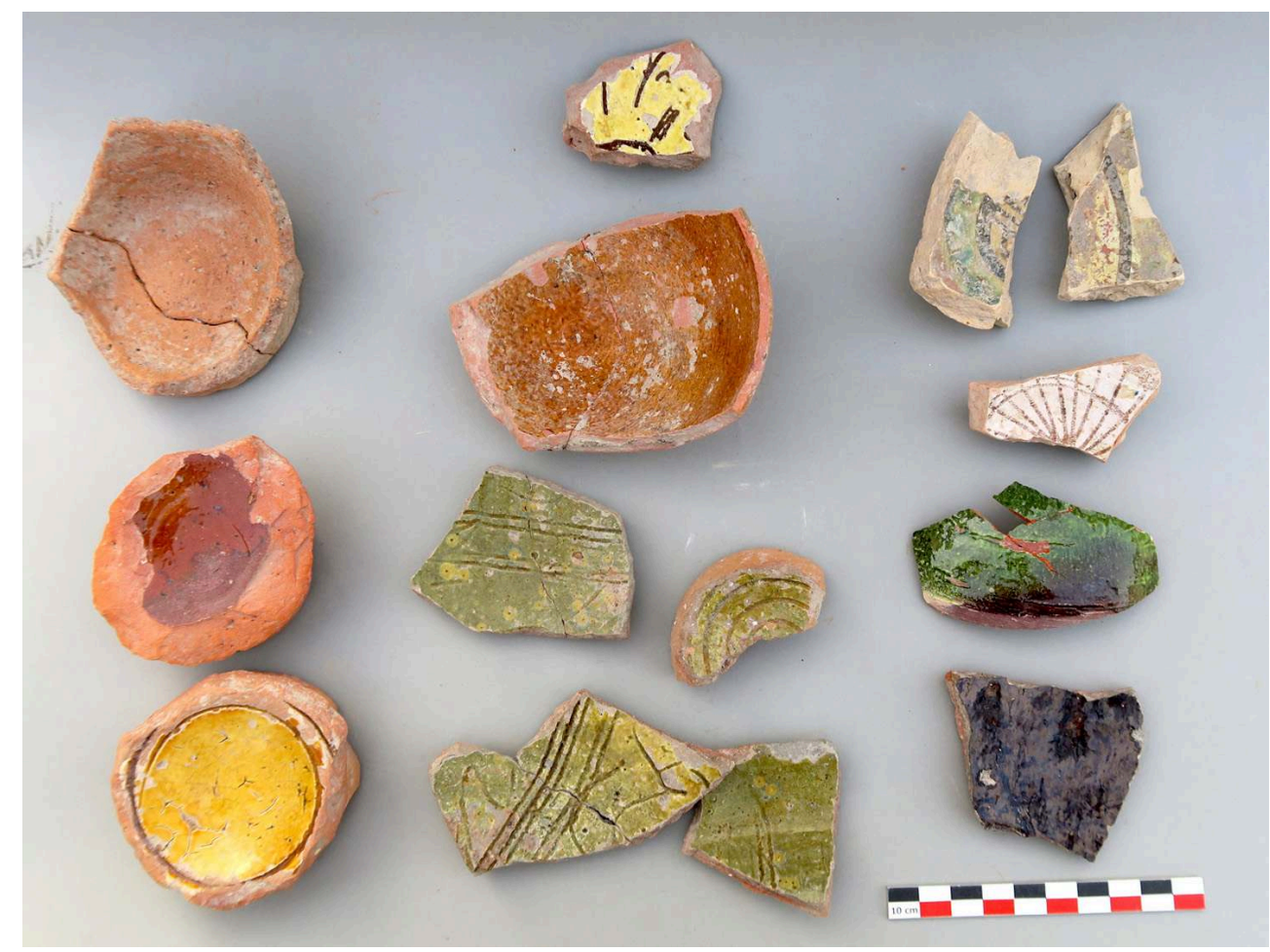

Cliché : L. de Barbarin.

Sous ce remblai, et sous le niveau de fondation de la structure hydraulique décrite précédemment, se trouvaient des niveaux argileux jaunâtres et compacts, entrecoupés de petites fosses et de foyers. Le mobilier assez abondant trouvé dans ces couches, homogène et sans intrusions récentes, permet d'échelonner ces niveaux entre l'Helladique Ancien et Moyen. La céramique comprend notamment des pâtes claires à décor mat (matt-painted ware) et de la céramique dite minyenne grise. Ces niveaux helladiques ont pu être suivis jusqu'au substrat, dont les irrégularités étaient bouchées par un sédiment brun-jaune, contenant peu de mobilier. L'interprétation de ce secteur à ces périodes anciennes est en revanche délicate, en raison de l'absence de structures permettant un diagnostic clair. La seule structure associée à l'horizon helladique, en fait, est un mur, USM 1025, au nord du sondage ; ce mur est fait de moellons de grande taille, sommairement agencés; le mur USM PK2, nettement plus récent, est fondé directement sur ces vestiges helladiques (fig. 6). Signalons enfin que ce mur USM 1025 était bordé au sud par une couche argileuse compacte, qui pourrait provenir de l'effondrement et de la décomposition d'une élévation en briques crues ou en pisé. 
Fig. 6. Fondation des murs USM PK2 et USM 1025.

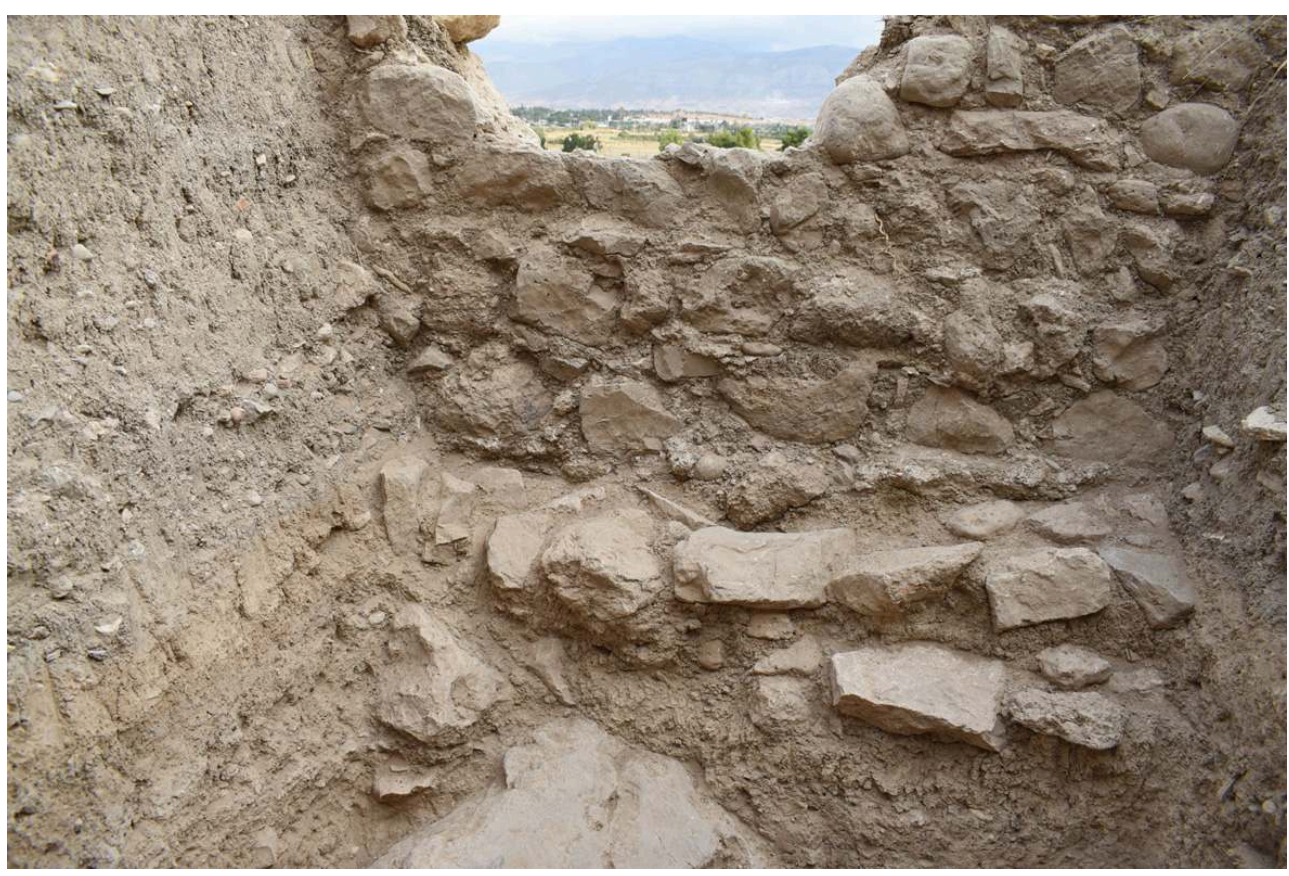

Cliché : A. Peeters.

16 Le mur du kastro à proprement parler, USM PK1 dans la terminologie de la campagne de 2018, est fondé directement sur le substrat rocheux (fig. 7); pour atteindre ce dernier, une tranchée de fondation avait été creusée dans les niveaux helladiques. Cette tranchée n'a cependant pas permis de dater la construction du kastro avec précision; elle était en effet entièrement remplie avec du mortier, et nous n'avons donc pas pu prélever de matériel datant dans le comblement de cette tranchée (fig. 8). 
Fig. 7. Vue générale du mur USM PK1.

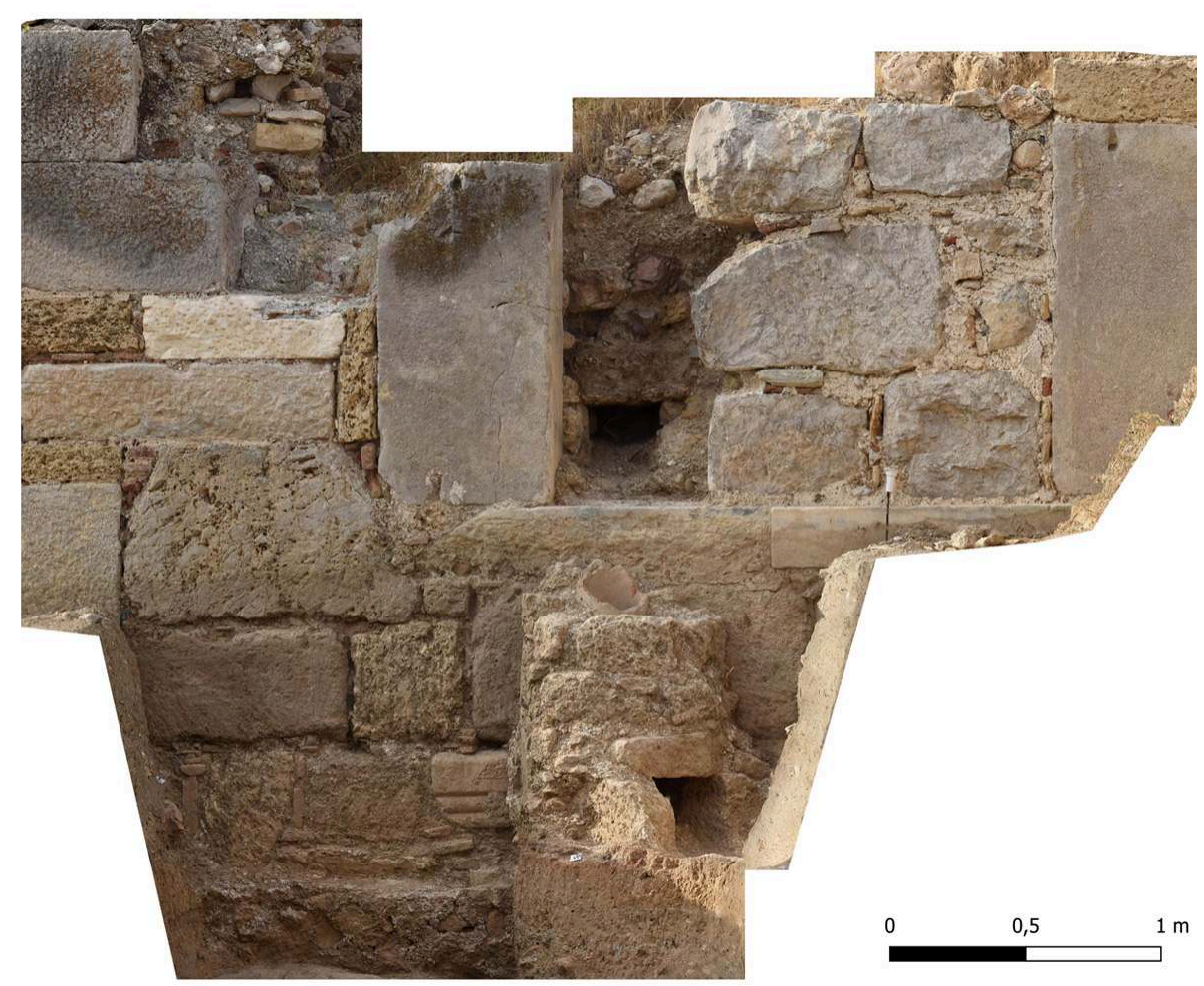

Orthophotographie et DAO : A. Peeters.

Fig. 8. Fondation du mur USM PK1.

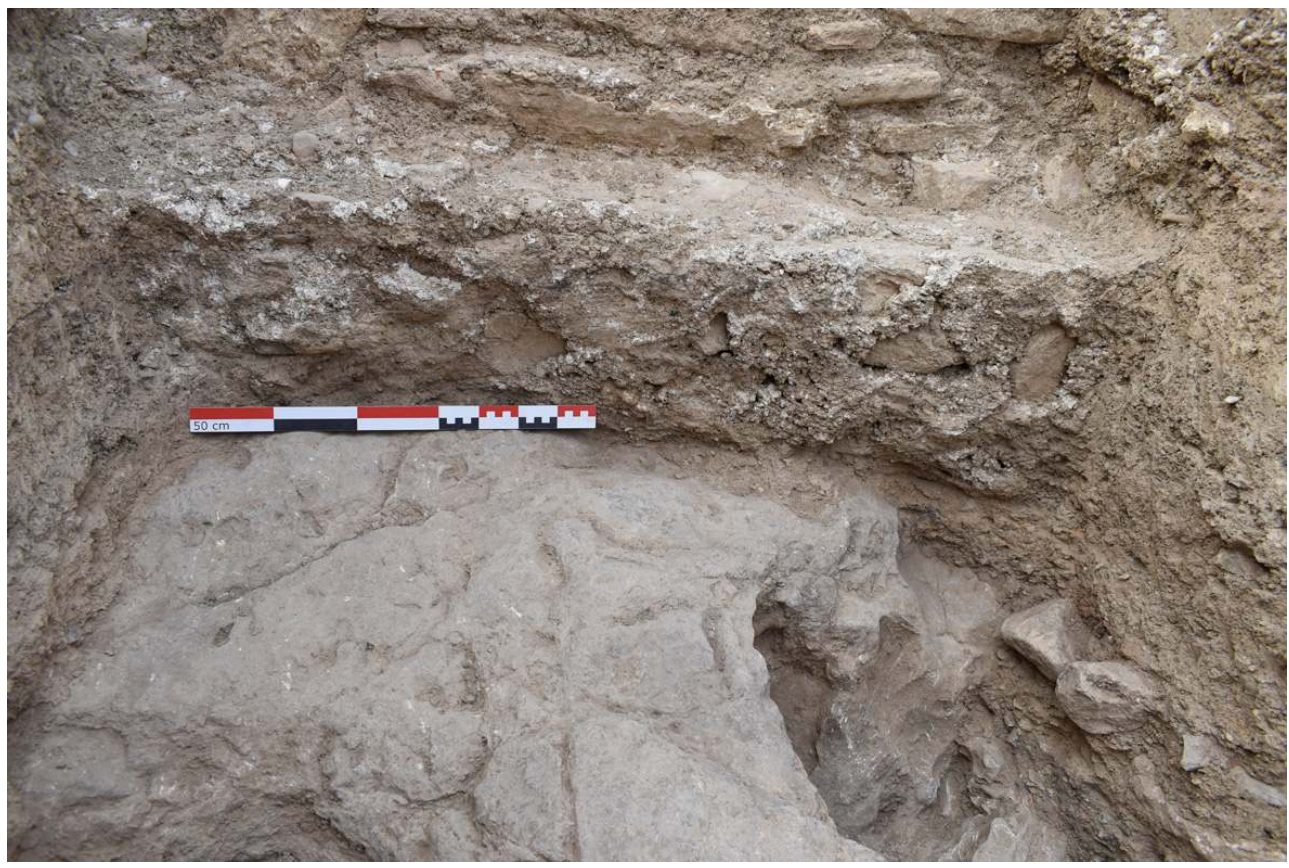

Cliché : A. Peeters. 


\section{PAL-6}

obtenus dans le secteur PAL-1: après un reste important de la couche de démolition et
une couche de remblai datable de l'époque médiévale ou ottomane, les niveaux
helladiques ont été atteints ; dans ce secteur, plusieurs fragments d'un même vase en
matt-painted ware ont été retrouvés contre les fondations du mur du kastro, et un tesson
de céramique à décor peint imitant les productions minoennes a également été mis au
jour (fig. 9).

Fig. 9. Fragment de céramique helladique du sondage PAL-6.

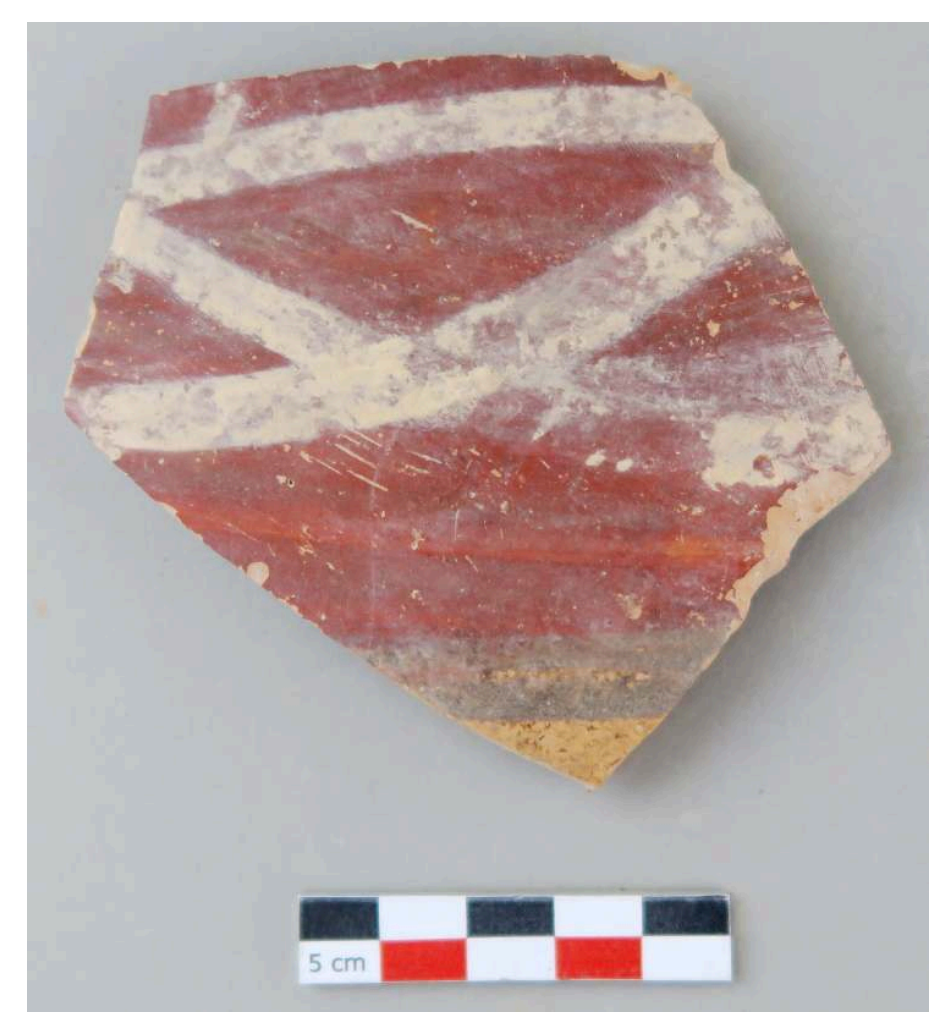

Cliché : L. de Barbarin.

Comme en PAL-1, la tranchée de fondation du mur du kastro, entièrement remplie avec du mortier, s'est avérée décevante. En revanche, on a pu constater que la tour nord avait été ajoutée lors d'une deuxième phase de construction. Ce fait, à vrai-dire, était déjà perceptible dès avant la fouille, en raison de l'absence de chaînage entre les murs de la tour et le mur du kastro. Au niveau des fondations, le mur de la tour vient s'appuyer simplement sur les premières assises en léger débord du mur du kastro et sur 
le mortier garnissant la tranchée de fondation. Fait notable, en revanche, la tranchée de fondation de la tour n'était pas remplie de mortier, témoignant de pratiques de construction différentes. Du matériel en connexion avec les fondations a ainsi pu être prélevé ; son analyse ultérieure devrait permettre de dater la fondation de la tour et, ce faisant, de proposer un terminus ante quem pour la construction du mur du kastro.

\section{Le kastro : conclusions préliminaires}

20 Les résultats des fouilles, en PAL-1 comme en PAL-6, permettent de proposer un schéma d'occupation à l'emplacement du kastro: le sommet de la colline de Palaiokastro a vu une première phase d'occupation sur un temps long, s'échelonnant entre l'Helladique Ancien et l'Helladique Moyen. La quasi-absence de structures identifiables rend l'interprétation de cette première occupation délicate.

21 Les couches supérieures, de même que le nettoyage de surface de 2018, ont livré des quantités importantes de matériel antique, échelonné entre l'époque géométrique et l'époque hellénistique. Le matériel archaïque est notamment très présent. Ce matériel résiduel est nécessairement lié à l'occupation du secteur aux époques concernées, sans qu'aucune structure en place ait été préservée. Il est probable que d'importants travaux de terrassement ont été menés en amont de l'établissement du kastro, conduisant à l'arasement complet des phases d'occupation antiques et à la récupération des matériaux de construction disponibles pour le kastro. Les niveaux helladiques ont cependant été préservés par les bâtisseurs, sans doute en raison de leur caractère compact et argileux ; ils offraient, en quelque sorte, une assise satisfaisante pour fonder les édifices et les niveaux de circulation plus récents.

Concernant l'occupation médiévale, il faut se résoudre à ne pouvoir dater que très grossièrement la phase de construction du kastro puisque, comme on l'a vu, la tranchée de fondation n'a pas livré de matériel exploitable. Cependant, la quasi-absence de matériel datant de l'antiquité tardive dans le remblai permet d'exclure cette période de façon presque certaine. Il faut donc plutôt dater le kastro de l'époque médiévale, au sens large; seule une analyse plus détaillée de la céramique, en particulier de la céramique glaçurée, permettra de préciser cette date.

Après sa construction, le kastro a en outre connu plusieurs aménagements ou réaménagements, identifiables par l'archéologie du bâti. Deux modifications peuvent ici être rappelées : l'édification de la tour nord, et le bouchage de l'ouverture située dans la partie ouest de PAL-1, incluant l'aménagement d'une structure d'écoulement des eaux; cette dernière venait sans doute remplacer l'écoulement direct par l'ouverture qui prévalait jusque-là. S'il est plus difficile encore de dater l'aménagement du mur de moellons USM PK2, parallèle au mur du kastro, il faut en tout cas y voir une autre phase de construction qui a vu l'établissement d'une terrasse au pied du mur du kastro, afin de favoriser la circulation ou d'adapter les défenses de celui-ci. Cette terrasse englobe la tour nord, et lui est donc postérieure. Pour ce dernier aménagement, une date ottomane est très probable, au vu de la céramique trouvée dans le remblai. 


\section{Le flanc est de la colline : le secteur PAL-4 (responsable : Jules Buffet)}

\section{Objectifs et implantation du sondage}

La localisation du secteur PAL-4 a été motivée par la présence de blocs de grand appareil affleurant dans la zone, qui laissaient entrevoir la présence de deux assises en place au moins (fig. 10). L'appareil pseudo-isodome, le soin apporté à l'ajustement des joints et le traitement brut de carrière de la face des blocs ne laissaient aucun doute sur le fait qu'il s'agissait là d'un mur de terrasse ou de fortification d'époque classique ou hellénistique, en place (USM 4080). Il a donc été décidé d'implanter un sondage, PAL-4, près de ces blocs, dans le but de dater et de comprendre cette structure affleurante et dans l'espoir de trouver des niveaux ou d'autres structures antiques en place. Dans ce cadre, les fouilles ont été menées dans un contexte particulièrement difficile, sur une pente dépassant par endroits les $30^{\circ}$. Cette configuration, ainsi que la faible épaisseur des niveaux au-dessus du substrat rocheux, a obligé à étendre progressivement le sondage initial, en fonction des découvertes et des endroits où la stratigraphie semblait mieux préservée, d'où la forme irrégulière du sondage final. La fouille du secteur PAL-4 a occupé les quatre semaines de la mission.

Fig. 10. Blocs en place au début de la fouille, après nettoyage.

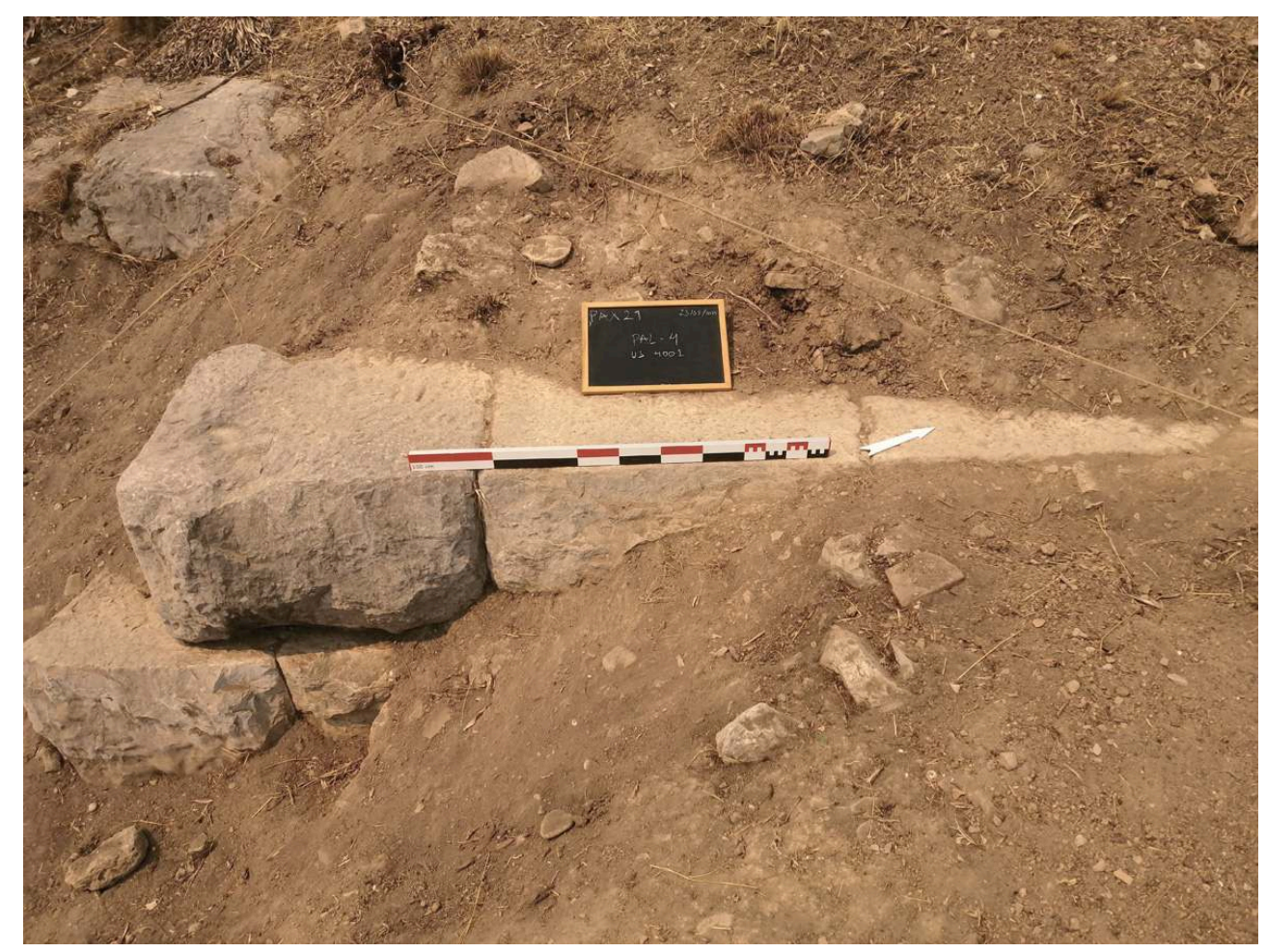

Cliché : J. Buffet. 


\section{Déroulement des fouilles et résultats : la tour hellénistique et l'occupation médiévale}

Les fouilles dans le secteur PAL-4 ont permis de mettre au jour au moins trois phases distinctes : des niveaux archaïques et classiques résiduels, dans lesquels était creusée une partie de la tranchée de fondation du mur USM 4080 ; une tour hellénistique (BAT 1) à laquelle appartenait le mur USM 4080, de forme carrée, de $8 \mathrm{~m}$ de côté ; enfin, une réoccupation plus tardive, médiévale ou ottomane, qui voit la construction sur le secteur de plusieurs murs de moellons grossiers (USM 4082, 4083, 4084 et 4085) d'interprétation délicate. À cette phase il faut également rattacher une tombe découverte au nord-ouest du secteur, qui contenait les ossements de trois individus au moins (fig. 11).

Fig. 11. Vue générale du secteur PAL-4 en fin de fouilles.

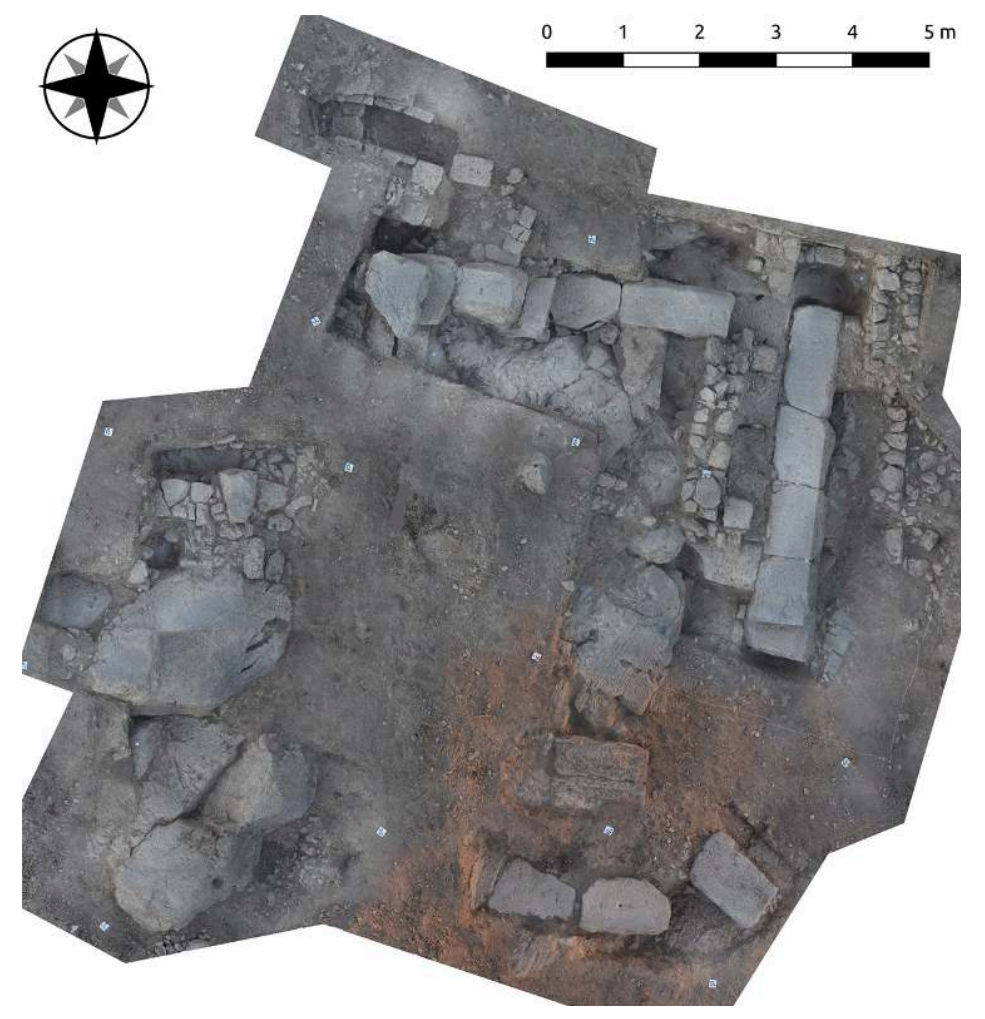

Orthophotographie : A. Peeters, DAO : Th. Lucas.

La découverte principale du secteur PAL-4 reste la tour BAT 1, dont la partie du mur USM 4080 visible avant le début des opérations constitue le front est, orienté nord-sud. La fouille a permis de dégager le flanc nord de cette tour, dont les blocs, étagés pour suivre le niveau de la pente, sont disposés sur quatre niveaux successifs. Le bloc qui formait l'angle entre le flanc nord et le front est de la tour est manquant, mais son négatif est bien visible dans le substrat, taillé pour le recevoir. L'angle sud-est est plus problématique, le front de la tour n'étant pas préservé sur toute sa longueur. Un bloc, USM 4087, posé en place sur le substrat taillé, semble correspondre à l'angle de la tour, mais son parement n'est pas aligné avec USM 4080. Il est possible qu'à cet endroit, l'angle de la tour ait été légèrement tronqué. Le front sud de la tour est bien identifiable grâce à la présence d'un autre bloc, USM 4096, lui aussi en place sur le 
substrat taillé ; il laisse présager un front sud strictement parallèle au flanc nord. Les deux derniers angles de la tour, au sud-ouest et au nord-ouest, n'ont pu être identifiés formellement par la présence de blocs en place; mais l'angle sud-ouest est à restituer de façon certaine dans une zone du secteur où le substrat a été taillé pour recevoir les blocs du flanc ouest de la tour. L'angle nord-ouest, quant à lui, était situé au-delà des limites de la fouille.

Grâce aux données de la fouille, il est possible de restituer sommairement le plan de cette tour, qui formait un carré presque parfait de $8 \mathrm{~m}$ de côté environ (fig. 12). Le départ de la courtine a pu être identifié sur le flanc ouest de la tour : à cet endroit, le négatif des blocs dans le substrat permet de restituer un mur de courtine perpendiculaire à celui de la tour et de conclure que l'enceinte continuait sur le flanc sud de la colline de Palaiokastro; du substrat taillé et des blocs en place, visibles à plusieurs endroits sur les flancs sud et ouest de la colline, et notamment au ras du mur sud du kastro, confirment que la fortification continuait bien dans cette direction. Ce mur de courtine, au niveau du flanc de la tour, avait une épaisseur de 2 mètres environ. Depuis la tour, un autre mur de courtine partait en direction du nord: deux blocs en place trouvés au fond de la tombe, et présentant les mêmes caractéristiques techniques que les blocs de la tour, et notamment des trous de pince, correspondent vraisemblablement à ce départ de la courtine en direction du nord (fig. 13). La tour BAT 1 formait donc l'angle sud-est d'une fortification antique légèrement plus étendue que le kastro médiéval.

Fig. 12. En bleuté : emprise probable de la tour BAT-1.



Orthophotographie : A. Peeters, DAO : Th. Lucas. 
Fig. 13. Blocs du mur de courtine au fond de la tombe TB1.

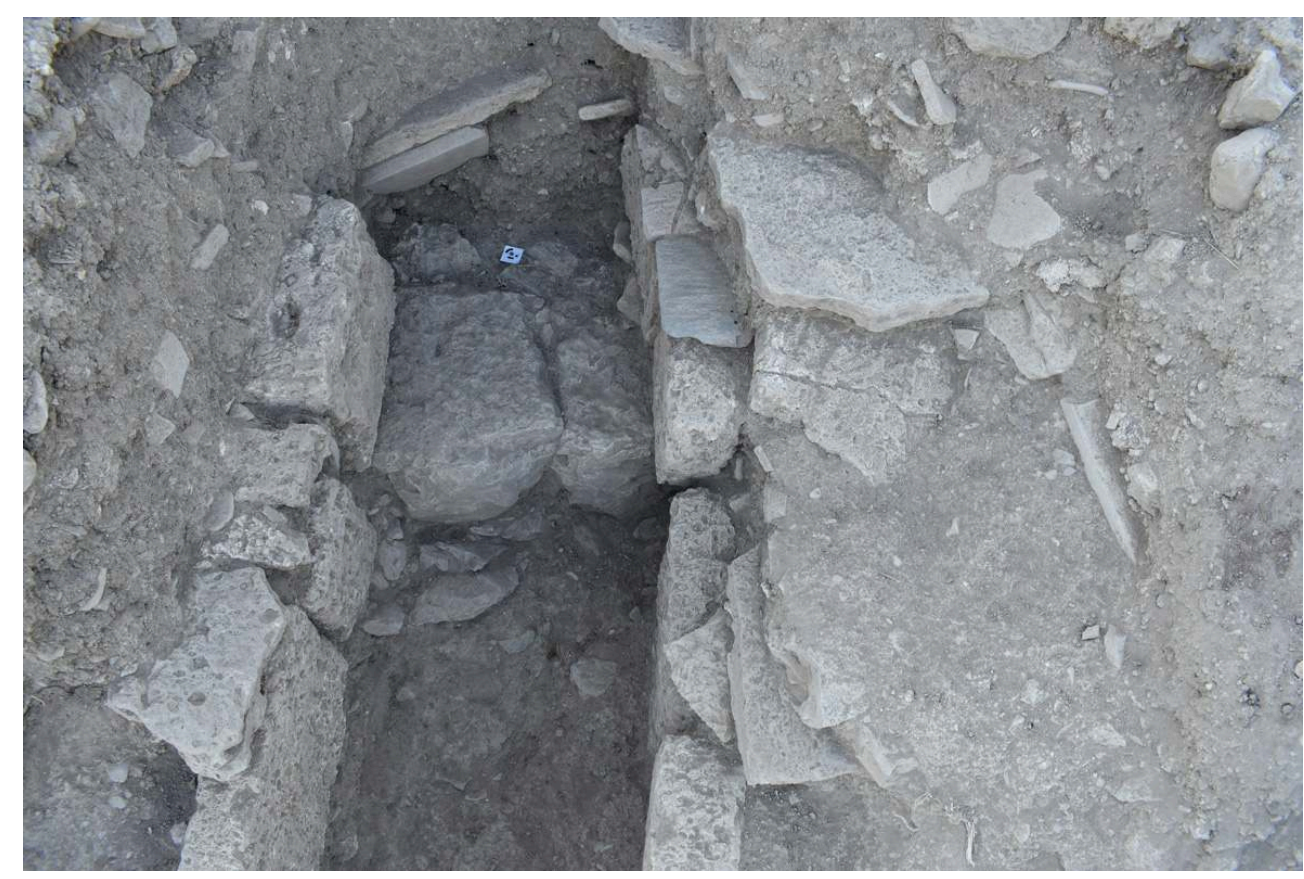

Cliché : A. Peeters.

Les blocs dégagés lors des fouilles correspondent essentiellement aux assises basses de la tour : celle-ci était fondée sur le substrat, largement entaillé et aplani pour recevoir les blocs. En particulier, le mur est de la tour devait commencer par plusieurs assises de fondation pour compenser la déclivité du terrain. Par endroits, les irrégularités du substrat ont été comblées avec les déchets de taille des blocs; ces couches de préparation se sont malheureusement révélées vierges de tout matériel permettant de dater la construction de la tour avec certitude. Sur le flanc nord, la tranchée de fondation de la tour a été en partie creusée dans des niveaux antérieurs, qui dataient, d'après la céramique trouvée sur place, de la fin $d_{u} \mathrm{VI}^{\mathrm{e}}$ ou du début du viècle. Les blocs sont soigneusement agencés et forment des assises apparemment régulières, sauf l'assise inférieure qui est adaptée à la pente. Les joints des blocs sont particulièrement soignés, et les faces de joint présentent de légers bandeaux d'anathyrose. Le lit d'attente porte fréquemment des trous de pince. Le parement des blocs est brut de carrière, et présente un bossage irrégulier fréquent dans les fortifications hellénistiques. La face arrière des blocs est seulement dégrossie. La tour, enfin, avait vraisemblablement une base pleine, comme du reste on pouvait s'y attendre sur une telle pente : la surface intérieure est irrégulière, et la face arrière non travaillée des blocs indique clairement qu'ils délimitaient un espace intérieur plein - d'ailleurs, les blocs visibles du flanc nord de la tour n'ont pas tous la même profondeur. On peut relever la présence, à l'arrière du mur USM 4080, de blocs de pôros de grandes dimensions appuyés contre le mur, qui servaient vraisemblablement à égaliser la surface à l'intérieur de la tour. Les blocs visibles appartenaient donc essentiellement aux substructures de la tour. En revanche, aucun élément ne permet de restituer l'élévation, les ouvertures ou la couverture de la tour; il est probable qu'une partie importante des blocs ait été remployée lors de la construction du kastro, ou ait chuté en bas de la pente très prononcée à cet endroit. La réoccupation ultérieure du secteur a dû également être précédée d'un arasement des structures en place. 

en partie fondée sur des couches archaïques et classiques, ce qui donne un terminus post quem qui ne peut qu'être très vague. La fouille des couches directement liées à la construction de la tour, notamment des remblais d'égalisation du substrat, n'a livré presque aucun mobilier et il est peu probable que l'étude des rares tessons retrouvés permette de fixer la chronologie. Il faut alors se tourner pour la datation vers deux indices: les techniques de construction, et le matériel résiduel potentiellement lié à l'utilisation de la tour.

Il est toujours risqué de dater une fortification par des critères uniquement techniques, mais ceux-ci peuvent donner au moins une première idée, qui doit être confirmée par d'autres éléments. En l'occurrence, il faut relever que la tour, avec 8 mètres de côté, est de taille respectable; le parallèle le plus proche en Mégaride est sans aucun doute Aigosthènes, où les tours d'angle du front est mesurent 7,4 et 9 mètres de côté et datent de l'extrême fin $\mathrm{du}_{\mathrm{IV}}^{\mathrm{e}}$ siècle ou même de la première moitié du III siècle av. J.-C. Comme les tours d'Aigosthènes, la tour de Palaiokastro était certainement destinée à mettre en batterie de l'artillerie défensive : la position de la tour est idéale pour tenir en respect aussi bien le rivage que les approches orientales de la colline de Palaiokastro, tout en offrant une position de flanquement idéale sur les deux murs de courtine qui partaient de cet angle. Une telle tour d'artillerie, en retour, ne peut se comprendre qu'après la diffusion de la catapulte à torsion, après le milieu du IV siècle. Le type d'architecture, l'agencement et le travail des blocs, s'accorderaient bien avec une telle date.

31

l'absence d'éléments permettant de préciser l'architecture de la tour, le nombre et la forme de ses ouvertures, le nombre d'étages, etc., l'argument de la taille de l'emprise au sol est cependant faible à lui seul. Un autre élément chronologique pourrait venir de l'analyse détaillée du matériel du secteur; même hors d'un contexte clairement identifié, il devrait pouvoir renseigner sur la chronologie de l'occupation du secteur. En l'occurrence, certains éléments pourraient provenir de la phase d'occupation ou d'abandon de la tour; notamment, l'une des couches de remblai byzantin située à l'intérieur de la tour, à proximité du substrat, a livré un grey unguentarium complet (4023.1) (fig. 14). Il s'agit d'une forme typique de l'époque hellénistique. Vu sa position, ce vase pourrait provenir des niveaux de construction ou d'abandon de la tour. Plusieurs autres unités stratigraphiques dans le secteur PAL-4 ont livré des quantités appréciables de matériel hellénistique qui pourrait là encore être associé aux fortifications (fig. 15). Les éléments disponibles actuellement laissent donc penser qu'une date hellénistique est encore l'hypothèse la plus probable pour la tour. Une étude détaillée de l'ensemble du mobilier du secteur est cependant nécessaire pour se faire une idée définitive et pour affiner la chronologie. 
Fig. 14. Grey unguentarium 4023.1.

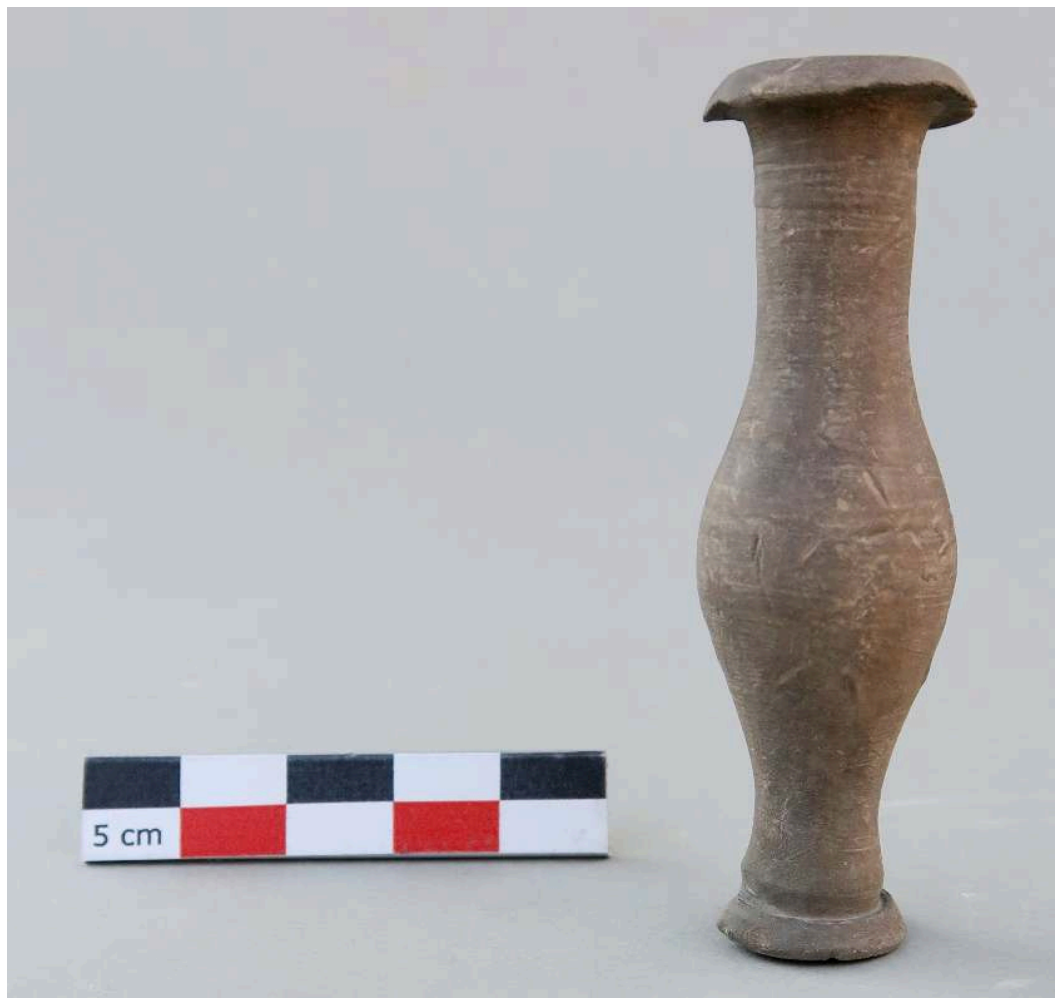

Cliché : L. de Barbarin.

Fig. 15. Sélection de céramique hellénistique du secteur PAL-4.

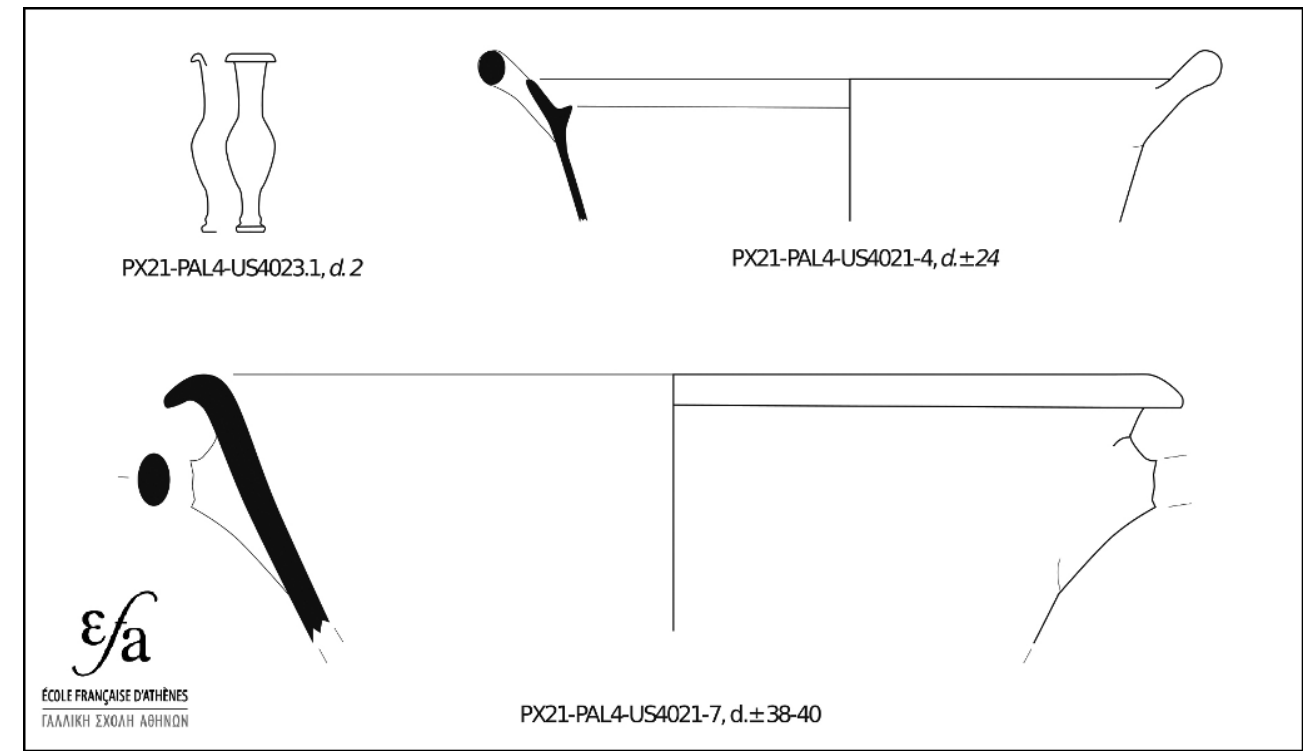

DAO : L. de Barbarin.

Le dégagement de la tour et de ses abords a également permis de mettre au jour des vestiges relevant d'une occupation postérieure du secteur ; plusieurs murs de moellons assez grossiers, très mal préservés, sont venus s'installer sur les vestiges de la tour ou à proximité ; on note également la présence d'un tambour de colonne lisse en marbre, à dater assurément à l'époque médiévale. Un premier mur, parallèle au front est de la 
tour, a été notamment édifié à l'extérieur de celle-ci ; d'après la céramique trouvée au contact de ce mur, il pourrait dater de l'antiquité tardive. À l'époque médiévale ou ottomane, d'autres murs ont été fondés sur l'emplacement même de la tour, les blocs restants du mur USM 4080 dessinant une petite terrasse facile à utiliser. Un petit bâtiment à la fonction indéterminée, BAT 2, se laisse deviner au niveau de l'angle nord-est de la tour ; son plan est très incomplet et l'élévation se résume à trois assises de mauvais moellons, ce qui limite d'emblée toute tentative d'interprétation. L'intérieur de la tour a également livré des niveaux d'occupation byzantins ou ottomans, incluant de la céramique culinaire, un récipient de stockage et plusieurs objets pseudo-sphérique percés, en pierre, qui pourraient être des poids de filet de pêche (fig. 16). L'érosion et le colluvionnement lié à la pente dans le secteur ont malheureusement rendu ces niveaux très isolés, de sorte qu'il est impossible de les mettre en relation avec les structures environnantes, et notamment BAT 2.

Fig. 16. Sélection d'objets du secteur PAL-4.

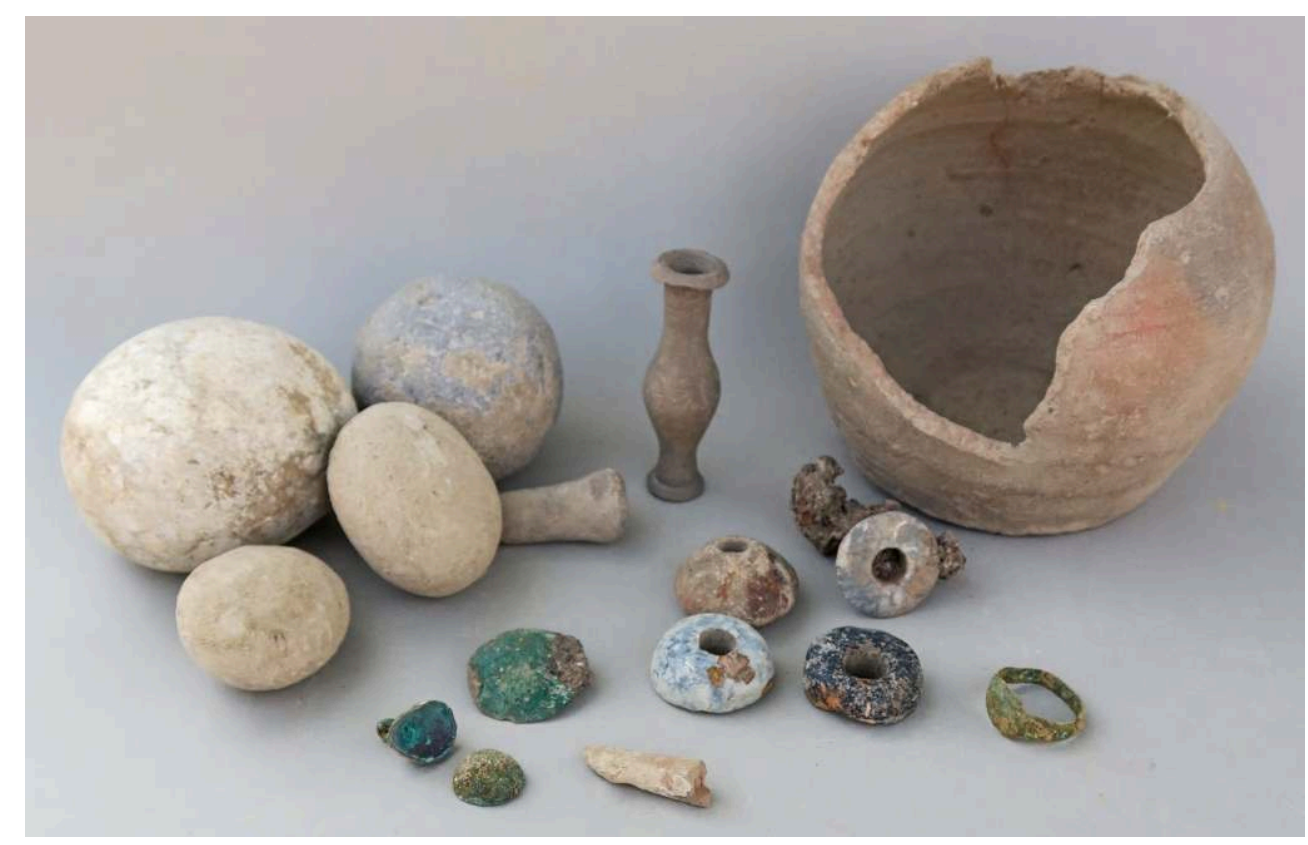

Cliché : L. de Barbarin.

Le long du parement externe du mur USM 4080, enfin, des contextes très perturbés, incluant du matériel très récent, ont été identifiés; une tranchée, dont le comblement inclut un étui de munition de la Seconde Guerre Mondiale et divers éléments métalliques modernes, court le long du mur USM 4080; il doit s'agir soit d'une perturbation liée au colluvionnement, qui a suivi le chemin le plus naturel en contournant les vestiges, soit d'un creusement lié à la construction de la batterie de la Seconde Guerre Mondiale, dont l'une des pièces se situe juste en haut du secteur, et qui comprenait également un bunker en béton armé en contrebas; il devait y avoir un sentier permettant d'assurer la circulation entre ces différents éléments, qui a pu prendre la forme d'une tranchée. 


\section{La tombe TB1 (responsable de la fouille : Sylvain Grosfilley)}

Au nord du secteur, les travaux d'extension du sondage pour comprendre la relation de la tour avec l'espace avoisinant ont conduit à la découverte d'une tombe, orientée est-ouest. La tombe en elle-même était couverte d'une couche de démolition épaisse, incluant des blocs de calcaire coquillier stuqués et une inscription funéraire d'époque hellénistique ou romaine (voir annexe). Le poids de ces blocs a conduit à la rupture des dalles de couvertures de la tombe - celles-ci, très hétérogènes, incluaient également une tuile. La dalle située le plus à l'est était manquante, de sorte qu'une partie des os se sont trouvés directement exposés, et nous n'avons eu d'autre choix que de fouiller entièrement la tombe, au prix d'une extension du sondage. En raison de la pente, il a été décidé de limiter cette extension au minimum : une extension plus grande aurait nécessité d'enlever à l'ouest une épaisseur de sédiment de plus d'un mètre, et par conséquent d'aménager les bermes pour les sécuriser. La fouille de la tombe a été confiée à l'archéologue Sylvain Grosfilley, membre de la mission le plus expérimenté pour ce type de fouille, qui a mené le travail dans ces conditions délicates. Pour l'enregistrement des données, nous avons bénéficié des conseils précieux de Dominique Henry-Gambier et de Reine-Marie Bérard (CNRS). Un dessin a été réalisé avant d'enlever la couverture de la tombe, par l'architecte Konstantina Theodoropoulou. Une fois la fouille entamée, il a été décidé de travailler par passes, en réalisant entre chaque passe importante une orthophotographie de l'ensemble de la tombe. Les orthophotographies ont été assemblées et calculées par Anthony Peeters. Les ensembles anatomiques en connexion et les os isolés ont été prélevés séparément, en relevant pour chaque os la face d'apparition, le pendage et l'orientation. En raison de la complexité de l'inhumation, la fouille de l'ensemble a pris deux semaines, c'est-à-dire l'intégralité du temps disponible à compter de la découverte des premiers os.

La sépulture est délimitée, au nord et au sud, par deux parois (USM 4106 et 4107) constituées de blocs de calcaire coquillier de dimensions inégales, dont certains étaient stuqués - et donc probablement en remploi - et de plaques étroites de schiste et de marbre, également en remploi. Signalons que la paroi sud est strictement alignée avec de petits blocs visibles plus bas dans la berme nord du sondage, de sorte qu'il n'est pas exclu qu'il s'agisse d'une canalisation, utilisée dans un second temps pour déposer des défunts; à l'appui de cette hypothèse, signalons que nous n'avons pu identifier formellement la limite ouest de la tombe, et que la limite hypothétique, à l'est, n'est représentée que par un petit bloc, qui ne ferme pas complètement la sépulture. Le fond de la tombe n'était en outre pas régulier ni plan, ce qui s'accorderait bien avec une canalisation échelonnée en paliers dans la pente. La dalle de couverture orientale, manquante, a sans doute été enlevée à une date postérieure, ou bien elle a simplement chu dans la pente de même, peut-être, qu'une partie des os. Le reste de la couverture, ainsi qu'on l'a vu, s'était effondré à l'intérieur de la tombe, endommageant partiellement les os : l'une des dalles en pierre, ainsi que la tuile, s'étaient brisées par le milieu, tandis que la dalle la plus à l'est avait simplement basculé obliquement dans la tombe. 
La tombe contenait les restes d'au moins quatre corps, numérotés par ordre d'identification (fig. 17) - en rétablissant l'ordre chronologique des dépôts, on peut décrire la situation comme il suit :

- Un premier défunt ( $\mathrm{S} 1$ ) était en réduction le long des parois de la tombe; son crâne se trouvait à l'extrémité sud-ouest de la tombe, le bassin au nord-ouest, les os des jambes se trouvaient contre les parois nord et sud de la tombe. Il est probable que cette réduction ait inclus les os d'un autre squelette très incomplet (S4), car plusieurs os dont les épiphyses n'étaient pas soudées ont été retrouvés dans cet ensemble, alors que le crâne identifié comme celui de $\mathrm{S} 1$ présentait les traits d'un adulte déjà assez avancé en âge : les sutures osseuses étaient solides, et les dents étaient dans un état de délabrement avancé peu compatible avec un individu jeune.

- Un deuxième défunt(S2) était placé sur le dos au fond de la tombe, en connexion anatomique, la tête orientée vers l'ouest, les deux mains croisées sur le ventre. Il s'agit d'un individu de petite taille (moins de 1,60 m); les épiphyses non-soudées de certains os, l'absence des dents de sagesse sur la partie préservée de la mâchoire et la fragilité du crâne, très endommagé, laissent penser qu'il s'agissait d'un adolescent ou d'un jeune adulte. Le sexe du défunt n'a pu être déterminé, mais pourra peut-être l'être après étude plus détaillée des ossements. Le squelette était complet et dans sa position originelle, permettant l'identification de l'ensemble des os de façon sûre; signalons simplement qu'en raison de l'irrégularité du fond de la tombe, la colonne vertébrale avait légèrement perdu sa continuité au-dessus du bassin, et que la plupart des côtes du côté droit avaient été nettement rompues par la chute de la tuile de couverture. La tête, assez endommagée, était calée entre le crâne et le bassin de S1; son inclinaison laisse penser qu'elle était initialement soutenue par un support. On a retrouvé, entre les côtes, une petite agrafe en bronze, peut-être une épingle de linceul, et sur chaque pied une petite boucle circulaire en métal ; il s'agit dans doute des boucles des chaussures que portait le défunt (fig. 18). Ces éléments indiquent un dépôt soigneux.

- Enfin, un troisième individu, S3, avait été ajouté dans la tombe; il s'agit là de l'élément le plus intriguant de l'ensemble : seul le buste, bassin exclu, a été retrouvé, en connexion; il était placé sur les jambes de S2, et orienté en sens inverse, tête vers l'est. Ce buste était placé sur le ventre, les deux bras dans le dos, en connexion anatomique stricte, signe qu'il avait été déposé dans cet état et dans cette position. Les os longs des jambes étaient séparés du buste et du bassin. Le crâne était manquant - à l'exception peut-être de la mandibule inférieure, retrouvée entre les jambes de S2, mais il est possible qu'il ait été emporté par la chute de la dalle de couverture est. Il n'y a pour l'heure aucune explication satisfaisante à ce troisième dépôt, qui a tous les traits d'une inhumation hâtive et peu précautionneuse. 
Fig. 17. La tombe TB1 après ouverture et dégagement des os.

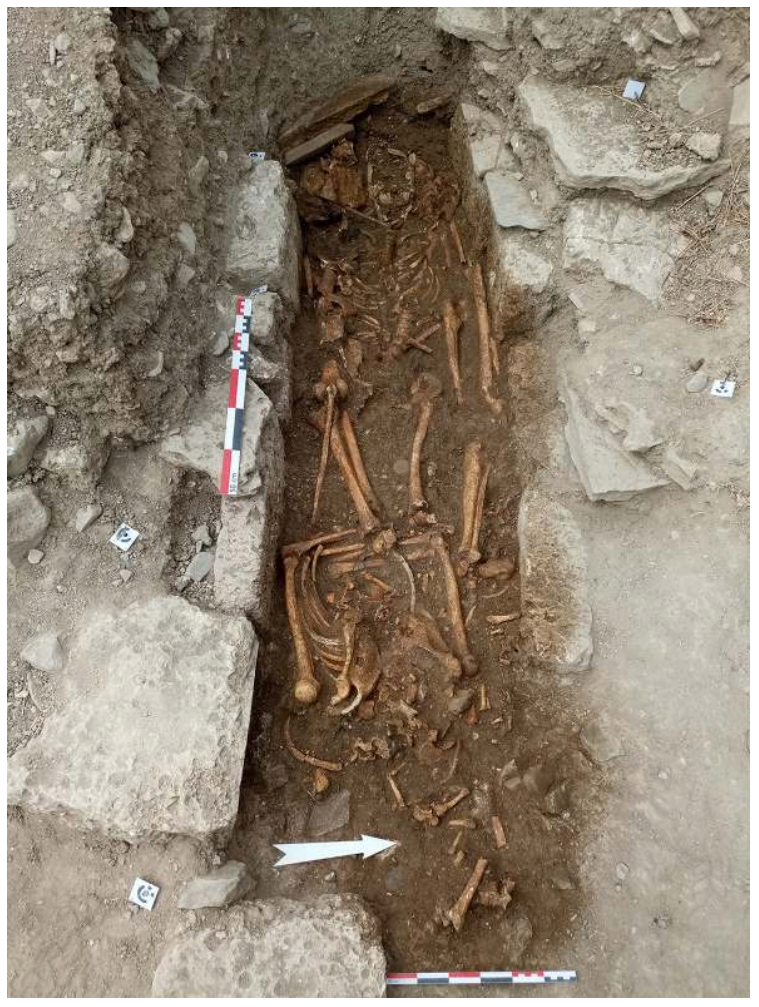

Cliché : S. Grosfilley.

Fig. 18. Objets métalliques trouvés sur le squelette S2.

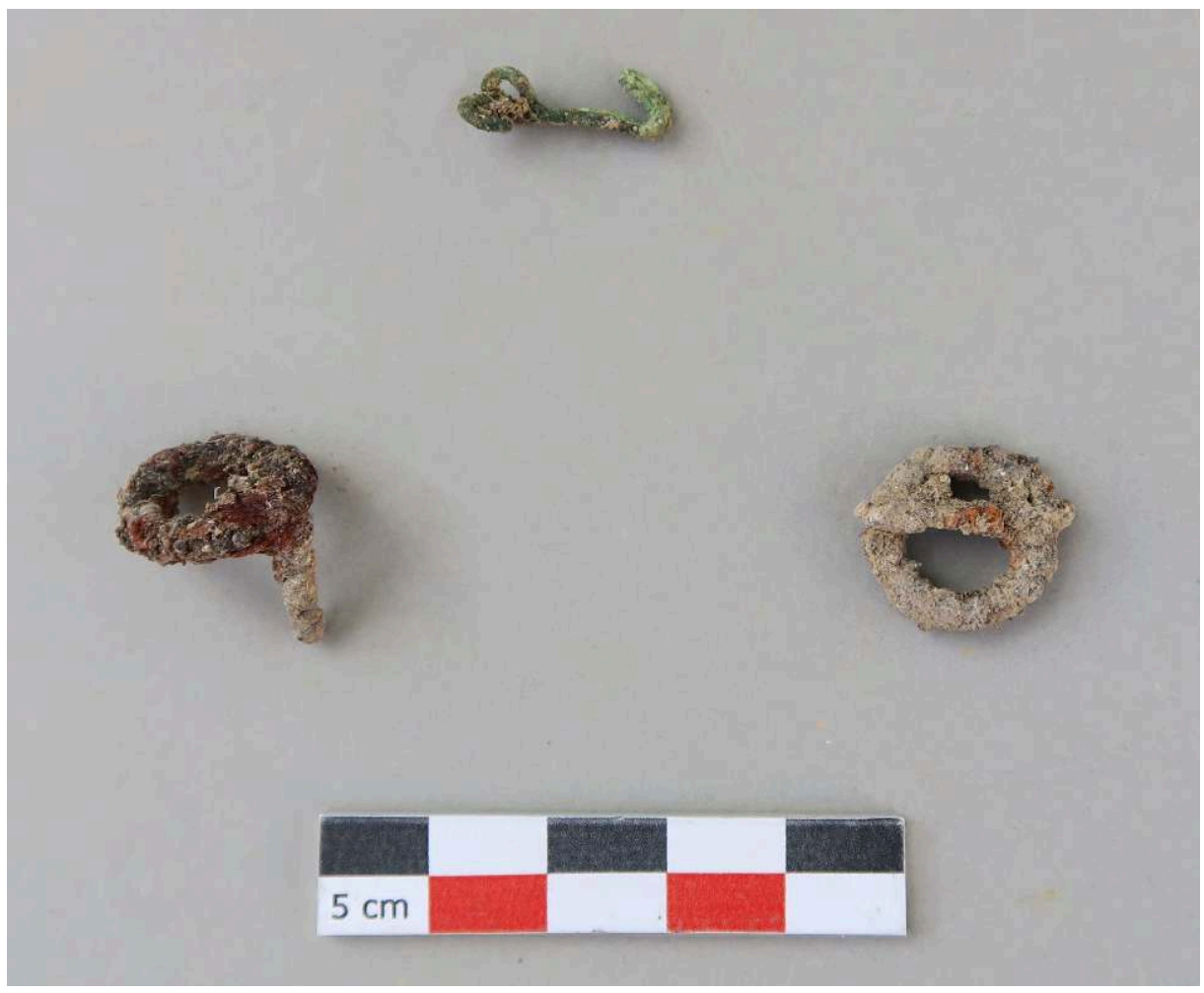

Cliché : L. de Barbarin. 
La fouille du sédiment remplissant la tombe n'a livré que très peu de mobilier ; outre les trois éléments métalliques présents sur le squelette $\mathrm{S} 2$, notons simplement la présence de rares tessons de céramique - incluant de la céramique glaçurée -, qui sont très certainement des intrusions et non du mobilier associé aux inhumations. Cette tombe doit cependant, en toute probabilité, être datée à la période médiévale : la tuile de couverture n'est certainement pas antique, et l'ensemble est nécessairement postérieur au démantèlement du rempart hellénistique. La fouille a été menée jusqu'au niveau de dépôt des défunts, constitué d'un sédiment brun-orangeâtre compact et caillouteux, présentant un fort pendage vers l'est, et coupé par deux blocs appartenant sans doute à la fortification hellénistique (voir supra). Par manque de temps, il n'a malheureusement pas été possible de pousser la fouille jusqu'au substrat rocheux dans ce secteur.

\section{Le secteur PAL-4 : conclusions et perspectives}

Le résultat principal du secteur PAL-4 reste l'identification d'une phase de fortifications hellénistiques sur la colline de Palaiokastro; cette découverte permet en retour de mieux comprendre certains blocs visibles sur le flanc opposé de la colline : plus que de murs de terrasse, il doit s'agir de la continuité de cette phase de fortifications. Le nettoyage des blocs visibles en surface a permis de confirmer cette impression. Le sommet de la colline était donc probablement ceint d'un rempart délimitant une aire légèrement plus vaste que celle du kastro ; Palaiokastro formait ainsi un point d'appui important pour défendre Nisaia, complémentaire des fortifications explorées sur Agios Georgios en 2017 et 2018 : le site portuaire était comme encadré de part et d'autre par des collines fortifiées, formant deux acropoles. Ce constat permet de revenir sur l'interprétation qu'il faut donner à la colline de Palaiokastro, qui n'était certainement pas l'̂̂le appelée Minôa par Thucydide ${ }^{3}$, quoi qu'on ait pu en dire. Palaiokastro faisait indéniablement partie de Nisaia et du réseau de défenses côtières associé à cet établissement. Signalons sur ce point que le seul tronçon correctement documenté de la jambe ouest des Longs-Murs de Mégare se trouve nettement à l'ouest de Palaiokastro, signe que la colline était englobée dans les fortifications ${ }^{4}$.

Les fouilles ont montré qu'il restait quelque chose de cette phase de construction hellénistique, dont l'état de préservation est malheureusement peu satisfaisant. Des recherches futures sur le site seront en tout cas nécessaires pour mieux comprendre cet état de l'occupation du site.

\section{Le flanc nord de la colline : le secteur PAL-5 (responsable : Louis Pirat)}

\section{Objectifs et implantation du sondage}

Le versant nord de la colline de Palaiokastro est caractérisé par une terrasse plane située en avant du mur USM PK2 et parallèle à celui-ci, large de 7 à 10 mètres; au-delà de celle-ci, la pente descend de façon assez abrupte jusqu'à la plaine en contrebas. Cette terrasse constituait un emplacement idéal pour tester la stratigraphie. Cependant, la surface de cette terrasse est fortement perturbée à son extrémité orientale, située en contrebas du sondage PAL-1. En son centre, elle est percée d'une tranchée dont le fond est garni de béton armé, ce qui indique clairement qu'il s'agit de l'une des installations 
liées à la batterie antiaérienne de la Seconde Guerre Mondiale. La zone la plus propice à l'implantation d'un sondage était donc l'extrémité occidentale de cette terrasse, au nord-ouest de l'angle du kastro. L'enjeu était principalement de tester la stratigraphie sur cette terrasse, et de voir si elle conservait quelque chose des phases d'occupations antérieures à l'établissement du kastro.

41 Un premier sondage a été implanté sous la forme d'un rectangle de $3 \mathrm{~m}$ sur 2. Ce sondage a ensuite été étendu en fonction des découvertes. Par la suite, un deuxième sondage (PAL-5 - sondage 2) a été ouvert plus bas dans la pente, pour tester la stratigraphie au niveau de la rupture de pente. Ce sondage consistait en un simple rectangle de 3 mètres sur 4,5 , implanté perpendiculairement à la pente.

\section{PAL-5 - sondage 1 : déroulement et résultats}

Dans le sondage 1, l'occupation se résume à trois phases bien identifiées, avec des vestiges structurels: une phase de construction médiévale, avec un bâtiment présentant un mur en arc de cercle et un espace extérieur avec un foyer; des niveaux intermédiaires, comprenant notamment plusieurs fosses et une tranchée de récupération, eux aussi datables de la période médiévale ou de l'Antiquité tardive; enfin une importante installation de l'Helladique Ancien, incluant un niveau de démolition de brique crue scellant un espace d'habitat ou de stockage bien préservé.

Immédiatement sous la surface $\mathrm{du}$ sol, la fouille a révélé un mur (USM 5003) appartenant à un bâtiment d'époque médiévale, qui était sans doute d'une certaine importance. Ce mur se présente sous la forme d'un arc de cercle qui coupait le quart est du sondage. Il était contre-buté à l'intérieur par deux contreforts d'importance inégale, non liés à la structure du mur.

Contre le parement externe de ce mur se trouvait un foyer, constitué d'une zone semicirculaire pavée de tessons de tuiles et entouré de petits moellons. Cet aménagement était complété, au nord-ouest, par plusieurs blocs en calcaire coquillier en remploi alignés, posés à même le sol, qui faisaient vraisemblablement office de coupe-vent pour ce foyer. À l'angle sud du sondage, enfin, se trouvait une fosse emplie de petites pierres, près de laquelle se trouvait un chapiteau de pilastre ionique en marbre (fig. 19). 
Fig. 19. Vue générale de l'horizon d'occupation associé au mur USM 5003.

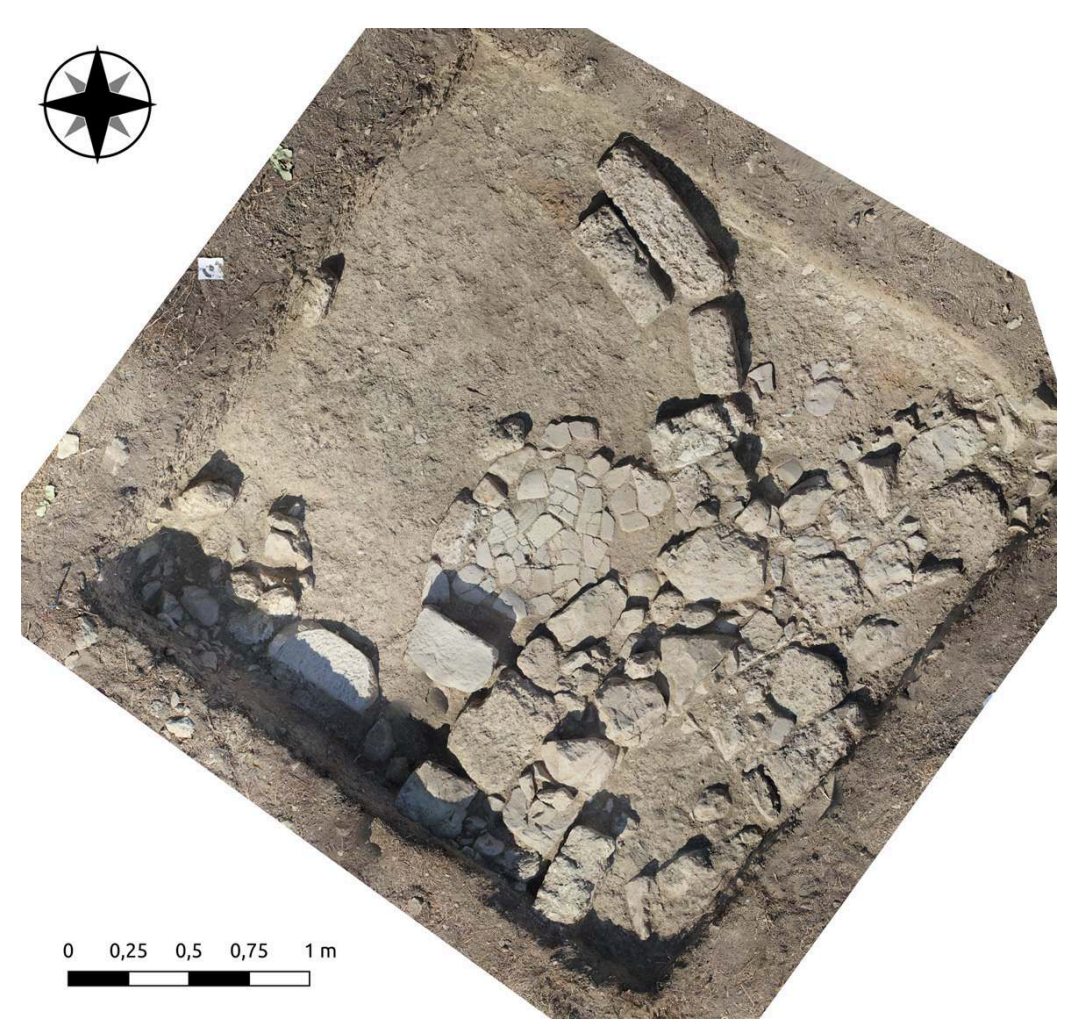

Orthophotographie : A. Peeters, DAO : Th. Lucas.

Devant ces premiers résultats, il a été décidé de profiter de l'espace extérieur pour pousser la fouille en profondeur. La fouille à l'intérieur du bâtiment a été limitée à une petite fenêtre entre les deux contreforts, pour examiner la fondation (SDINT 01) (fig. 20). Le niveau de sol n'a pu être trouvé, signe que l'ensemble des assises préservées de ce mur appartenait aux fondations de l'édifice. La fouille n'a révélé aucun mobilier qui permette d'identifier la fonction de l'édifice.

À l'extérieur du bâtiment, le sondage a été étendu, puis la fouille a repris essentiellement sur un rectangle de deux mètres sur trois localisé à l'angle ouest du sondage (SDINT 02). Dans cette zone, la fouille a été poursuivie sur une profondeur de deux mètres environ, jusqu'à la fin de la campagne; vu la profondeur, il n'a pas été possible d'atteindre le substrat: il aurait pour cela été nécessaire de sécuriser les bermes pour continuer la fouille, ce qui n'a pu se faire dans le temps de la campagne. 
Fig. 20. Le sondage SDINTO1 en fin de fouille.

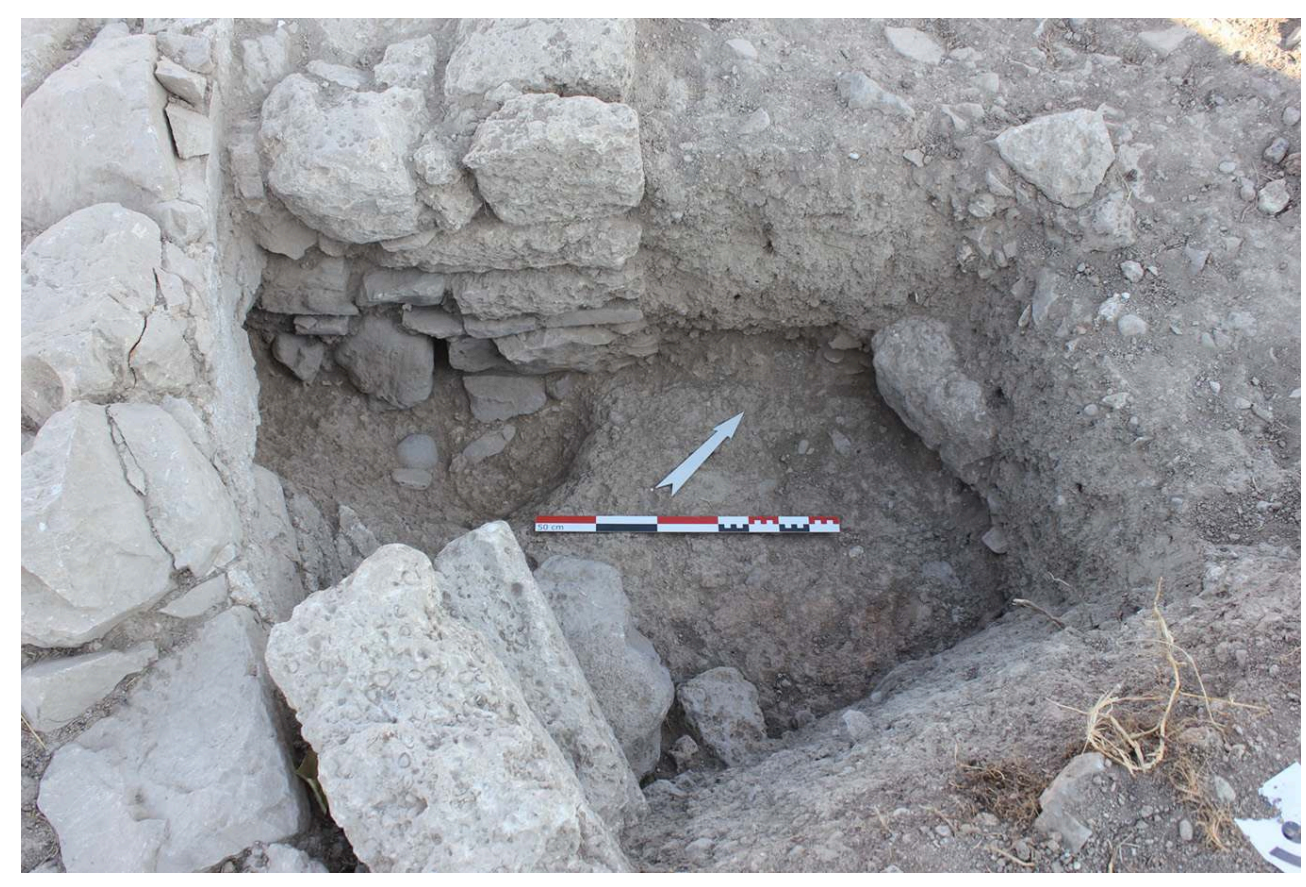

Cliché : L. Pirat.

47 En premier lieu, le foyer a été coupé en deux pour voir en coupe le niveau sur lequel il reposait. Sous ce foyer, une fosse a été identifiée et fouillée. Le comblement indique qu'elle doit être datée de la période médiévale. Un petit mur de moellons de mauvaise facture, très arasé, orienté nord-est - sud-ouest, a été identifié dans la partie occidentale du secteur. Il est à rattacher à la même phase; la présence d'un unique parement, au nord de ce mur, indique qu'il s'agissait là d'un petit ouvrage de terrassement.

Dans la partie du sondage menée en profondeur, un important pierrier (épaisseur: $0,75 \mathrm{~m}$ environ) a tout d'abord été mis au jour sous les niveaux assurément byzantins et dégagé. Le matériel y est majoritairement helladique, mais la présence résiduelle de céramique antique semble indiquer qu'il s'agit là d'un remblai plus tardif, ou du moins d'un niveau fortement remanié et perturbé. Ce niveau était d'ailleurs coupé par une importante tranchée au remplissage hétérogène, incluant des tessons antiques et byzantins.

À mesure de l'avancement du sondage, enfin, du matériel helladique a été découvert, en quantité de plus en plus importantes, incluant de la céramique (matt-painted ware et minyenne grise) et de petites lames d'obsidienne (fig. 21). La fouille du pierrier a révélé un horizon d'occupation helladique bien préservé, à rattacher très vraisemblablement à l'Helladique Ancien (ce diagnostic pourra être affiné lors de l'étude détaillée du mobilier). Cet horizon comprenait tout d'abord un niveau de destruction de briques crues, dont deux ont été prélevées (fig. 22). L'effondrement de ces briques avait scellé un espace intérieur, d'habitat ou de stockage. Des couches de cendres indiquent que la destruction s'est accompagnée d'un incendie ; parmi les cendres, on a trouvé deux éléments notables : un fragment de poutre calcinée bien préservé, de section circulaire, de $10 \mathrm{~cm}$ de diamètre environ, qui a été prélevé et pourra être analysé au $\mathrm{C} 14$; et un fuseau complet, incluant la fusaïole en terre cuite et la baguette, carbonisée, en 
plusieurs tronçons. Enfin, les fragments d'un grand pithos de stockage, archéologiquement complet, ont été prélevés (fig. 23). Celui-ci, pris au milieu de la couche de destruction et non sous elle, était vraisemblablement localisé sur un toit-terrasse ou à l'étage de l'édifice.

Fig. 21. Sélection de matériel lithique helladique du secteur PAL-5.

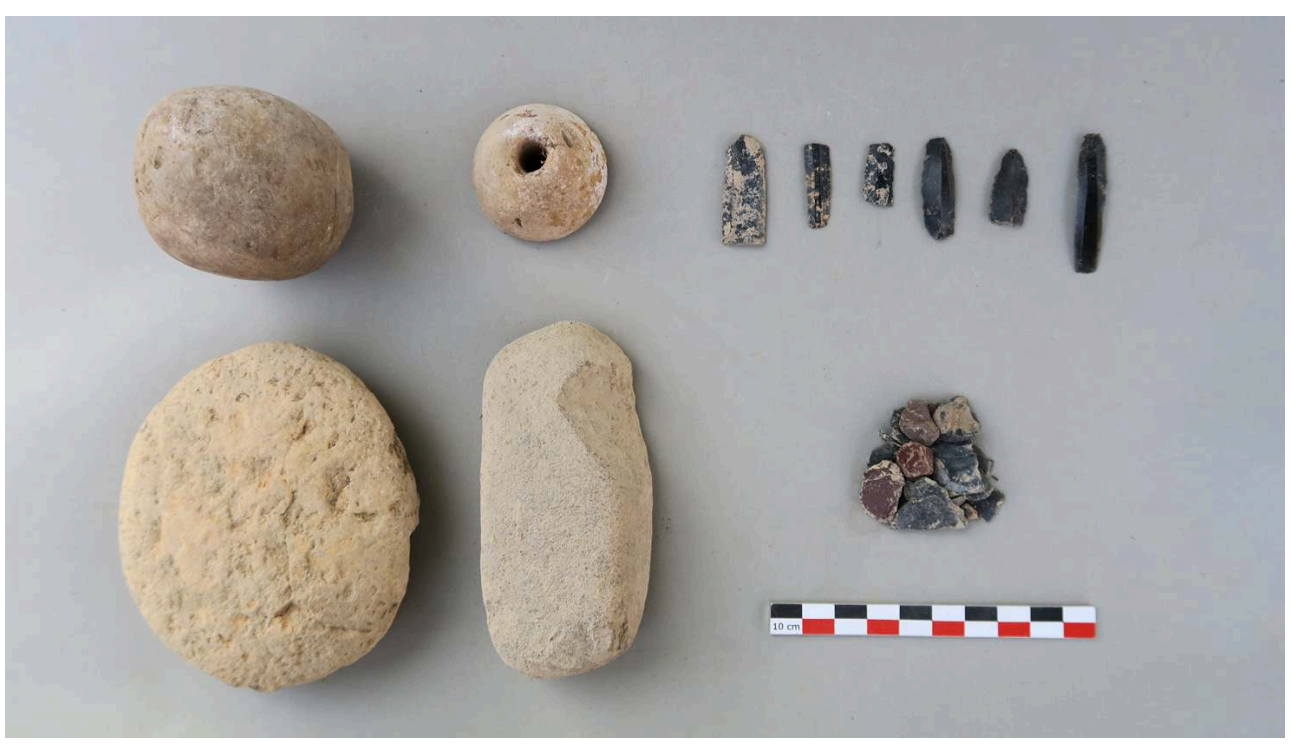

Cliché : L. de Barbarin.

Fig. 22. Vue générale du niveau de destruction US 5027.

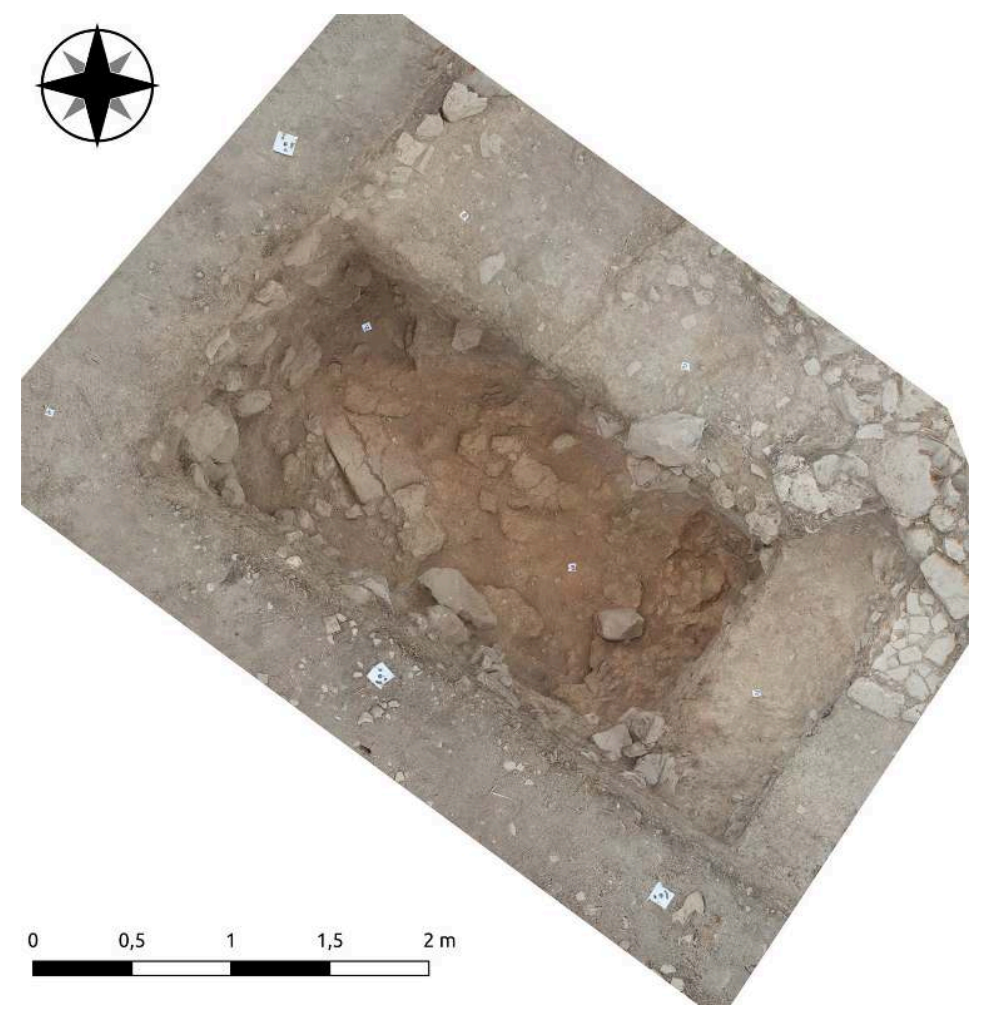

Orthophotographie : A. Peeters, DAO : Th. Lucas. 
Fig. 23. Fragments du pithos pris dans la couche de destruction.



Orthophotographie : A. Peeters, DAO : Th. Lucas.

50 Le niveau de sol correspondant à cet édifice a été dégagé sur l'ensemble de la surface ; par endroits, des moellons ont été retrouvés, qui provenaient vraisemblablement du socle en pierre sèche des murs de l'édifice - sur une surface cependant trop petite pour qu'on puisse en tirer des conclusions sur l'architecture de l'édifice. Par manque de temps, il n'a pas été possible de pousser la fouille plus loin que ce niveau de sol, mais le mobilier présent en surface a été prélevé; il devrait permettre de contribuer à la datation de la destruction de l'édifice.

\section{PAL-5 - sondage 2 : déroulement et résultats}

Pour compléter l'exploration de la terrasse parallèle au mur, il a été décidé, au cours de la mission, d'ouvrir un second sondage à l'endroit où la terrasse s'achève et où s'amorce la pente plus prononcée. En son point le plus profond, la fouille a été poussée sur une profondeur de 1,50 mètre (fig. 24). 
Fig. 24. La berme sud-ouest du sondage 2 en fin de fouille.

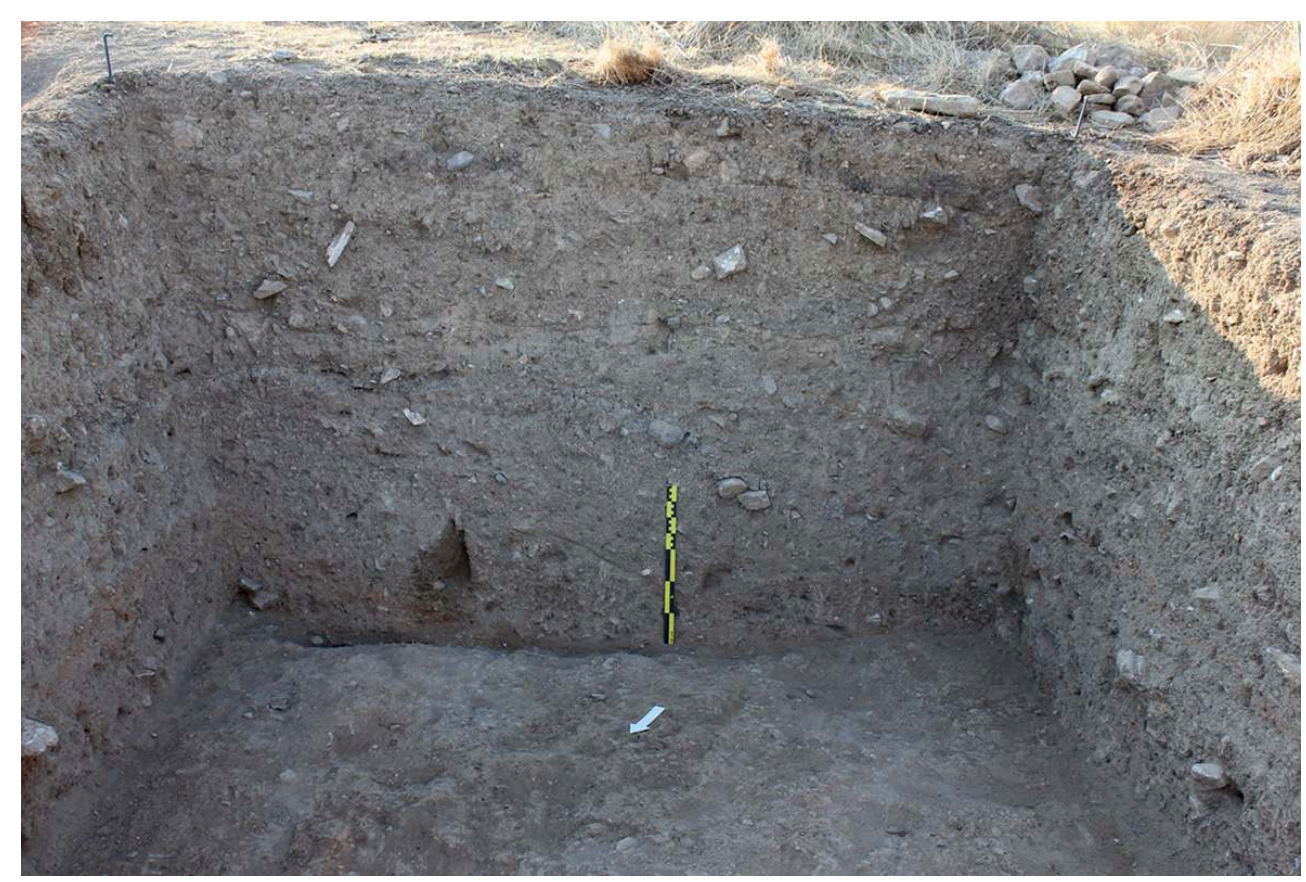

Cliché : L. Pirat.

52 Le sondage 2 n'a révélé absolument aucune structure, mais a permis de mieux comprendre l'histoire du site: les couches fouillées appartenaient à des niveaux de déblai, d'époque médiévale d'après le matériel le plus récent. Il a même été possible de suivre en coupe la micro-stratigraphie de ces déblais. Toutes les couches concernées ont livré un matériel abondant et très mélangé, incluant principalement de la céramique des époques mycénienne, géométrique, archaïque, et dans une moindre mesure, classique et hellénistique. Le matériel le plus récent, présent en faibles quantités, est à dater à l'époque médiévale (céramique glaçurée) (fig. 25 et 26). Le diagnostic de l'apothèque rejoint ici le diagnostic posé sur le terrain : malgré le nombre de fragments prélevé, quasiment aucun collage n'a pu être identifié, ce qui montre bien qu'il s'agit là de couches extrêmement perturbées. 
Fig. 25. Sélection de céramique de l'US 5026.

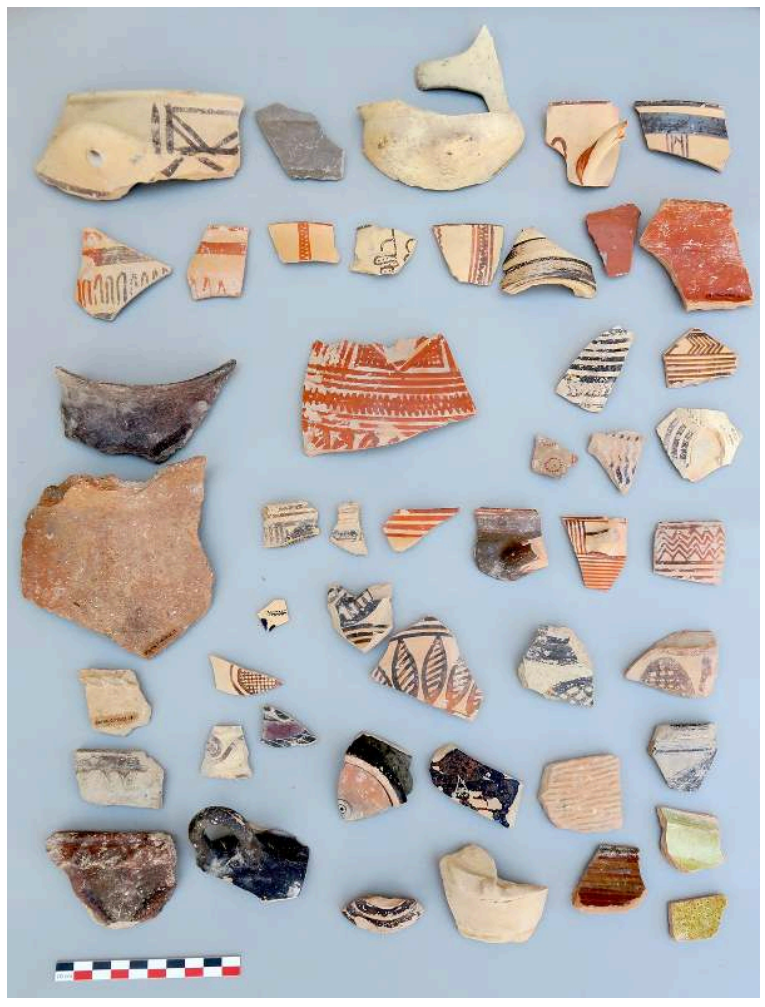

Cliché : L. de Barbarin.

Fig. 26. Sélection de céramique de l'US 5028.

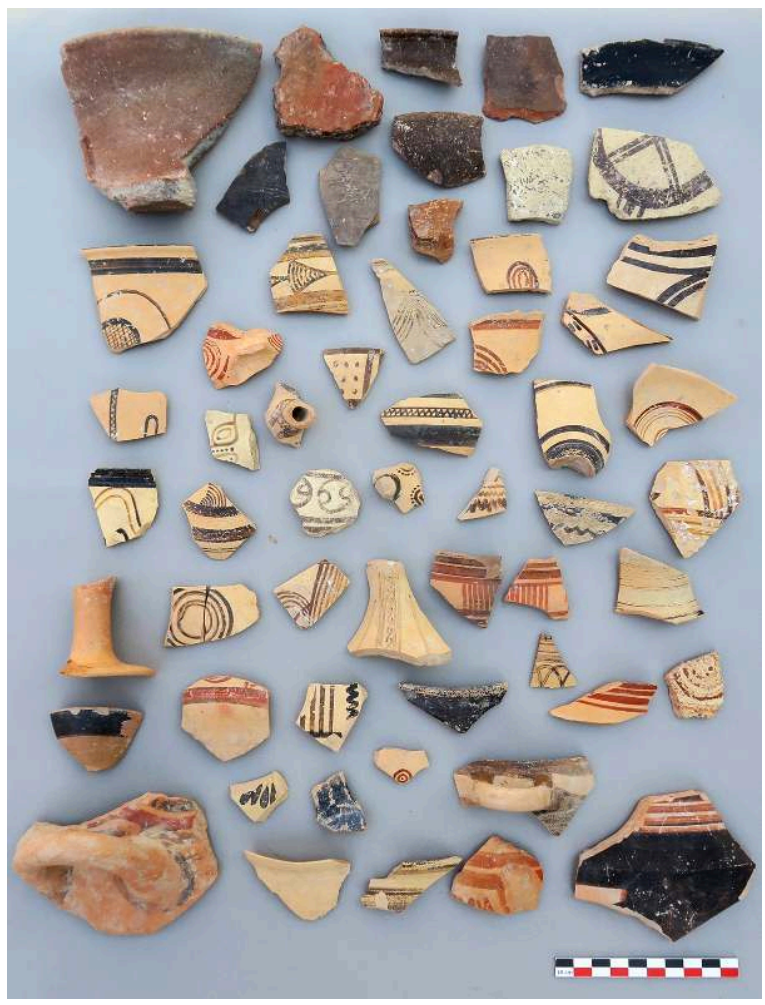

Cliché : L. de Barbarin. 
En retour, ce sondage permet d'expliquer un phénomène observé aussi bien en PAL-1 que dans le sondage 1 de PAL-5: le passage sans transition ou presque des couches tardives à l'horizon d'occupation helladique, alors même que le matériel résiduel des époques intermédiaires laisse supposer une occupation, notamment aux époques archaïque et classique. La conclusion atteinte pour PAL-1 doit probablement être étendue à l'ensemble du site: il semble bien que l'occupation médiévale ait été précédée d'un grand nettoyage du site qui a conduit à l'arasement des niveaux existants et à leur évacuation dans la pente. Comme dans PAL-1, l'occupation helladique a été préservée en raison de sa nature : la décomposition de brique crue a donné des niveaux compacts et stables, qui harmonisent naturellement la surface irrégulière du substrat.

Malgré les apparences, le résultat du sondage 2 est donc extrêmement positif: le mobilier trouvé dans ce secteur nous renseigne, plus sûrement qu'une prospection, sur les phases d'occupation principales du site, y compris celles qui n'ont pas été préservées dans la stratigraphie. Le mobilier datant de l'Helladique Récent et de la période géométrique, curieusement absent sur le reste de la fouille, est particulièrement bien représenté dans ces niveaux. On peut même formuler l'hypothèse que c'est durant le nettoyage du site que les bâtisseurs byzantins ont pu dégager et réutiliser les nombreux blocs architecturaux antiques qui forment l'essentiel de la structure du kastro. L'étude approfondie du matériel du sondage 2 sera donc capitale pour mieux comprendre l'histoire du site.

\section{Le secteur PAL-5 : conclusions et perspectives}

$\mathrm{Au}$ rang des résultats obtenus en PAL-5, on peut d'ores et déjà formuler deux enseignements principaux :

- Les niveaux helladiques sont particulièrement bien préservés sur la terrasse qui précède le versant nord de la colline de Palaiokastro. Ils représentent une phase déjà connue sur le site, mais jusqu'ici peu documentée. L'identification de céramique appartenant indubitablement à l'Helladique Ancien (en PAL-5 comme en PAL-1 et PAL-6) est en revanche une nouveauté.

- On a désormais la preuve que l'établissement du kastro a causé une perturbation majeure dans la stratigraphie du site : l'occupation médiévale a tout bonnement annihilé les niveaux correspondant aux phases d'occupation échelonnées entre l'époque mycénienne et l'époque hellénistique.

\section{Étude préliminaire et conditionnement du mobilier (responsable : Lou de Barbarin)}

En parallèle de la fouille, l'équipe d'apothèque a procédé à l'enregistrement et au conditionnement du mobilier, mais aussi à une étude préliminaire de la céramique, incluant la couverture photographique complète du mobilier, le diagnostic et les comptages préliminaires pour la datation de la plupart des unités stratigraphiques, ainsi que le dessin des vases les plus représentatifs de certaines US.

Au-delà de la nécessité de définir un horizon chronologique des sondages, ce travail a permis d'esquisser les premières problématiques de recherches en termes de céramique à Pachi et sur le territoire mégarien. L'approche quantitative et l'étude du 
mobilier amphorique d'Agios Georgios menées par Mikaël Pesenti en 2018 ont notamment mis en évidence l'importance des échanges en Mégaride aux $v^{\mathrm{e}}$ et $\mathrm{IV}^{\mathrm{e}} \mathrm{s}$. av. J.-C. Sur Palaiokastro, les différents secteurs offrent un large aperçu de la succession des périodes d'occupation de la colline, depuis le Bronze Ancien jusqu'à l'époque hellénistique pour l'antiquité, et de la période byzantine à l'époque moderne pour les époques plus récentes. Les nouveautés principales de la fouille de 2021, outre une belle quantité de mobilier géométrique et archaïque, résident dans l'identification des céramiques de l'Helladique Ancien et de la fin de l'Helladique Récent ainsi que dans l'observation de plusieurs hiatus dans l'occupation (début de l'Helladique Récent, haute période géométrique, époque romaine).

Cette première approche du mobilier permet dès à présent de rendre compte de l'importance du fond céramique que constitue le matériel des prospections et des sondages de Pachi, tant pour l'âge du Bronze que pour l'Antiquité et la période médiévale. Pour toutes ces périodes, le matériel est en effet abondant, bien conservé, et a livré des pièces parfois rares, toujours riches d'informations : par son caractère inédit pour la Mégaride, une étude du corpus de Pachi participerait à compléter une lacune très importante de l'histoire de la céramique protohistorique et grecque. Il faut en effet rappeler que la céramique de la Mégaride, importée comme locale, est à peu près parfaitement méconnue. Située entre Athènes et Corinthe, les deux références majeures en termes de céramique pour l'Antiquité, la production de Mégare brille par son absence dans l'histoire de la recherche sur les céramiques grecques en raison du manque de travaux publiés sur la question. Les importations céramiques, quant à elles, ont fait au mieux l'objet de mentions dans des rapports de fouilles ou des articles anciens ou ponctuels. Or, le mobilier céramique de Pachi, très abondant ${ }^{5}$, offre un fonds documentaire exceptionnel, tant en matériel amphorique, culinaire et commun qu'en céramique fine, permettant d'enrichir considérablement le dossier mégarien de l'âge du Bronze, de l'Antiquité et de la période médiévale.

Il reste naturellement beaucoup à faire : une campagne d'étude sera nécessaire, avant tout pour compléter l'enregistrement et l'étude des US mises au jour en fin de fouille en 2021, ensuite pour réaliser une étude plus approfondie de l'ensemble du mobilier, notamment celui issu des prospections et des fouilles d'Agios Georgios de 2017 et 2018.

La documentation correspondante a fait l'objet d'un rapport détaillé transmis à l'EFA, dont les conclusions chronologiques ont largement servi pour la rédaction du présent rapport.

\section{Conclusions générales de la campagne 2021 et perspectives pour de futurs travaux}

61 La fouille de 2021, d'une ampleur limitée, avait pour objectif de documenter scientifiquement l'occupation antique de Palaiokastro, en particulier les phases antérieures à l'époque médiévale. De ce point de vue, les résultats préliminaires les plus importants sont :

- L'identification et la description d'une phase de fortification hellénistique sur le site, en particulier en PAL-4 où l'on a pu identifier et décrire une tour d'angle de cette fortification.

- La mise en exergue, dans tous les secteurs, d'une importante modification de la morphologie de la colline à l'époque médiévale, qui a conduit à l'arasement et à la destruction quasi- 
complète des phases d'occupation antiques comprises entre l'Helladique Récent et la période hellénistique.

- L'identification d'une importante occupation datant de l'Helladique Ancien et Moyen, dont les niveaux, au moins sur le flanc nord de la colline, sont bien préservés. une vision plus solide du potentiel archéologique de la zone. On peut résumer les possibilités de travaux futurs à Palaiokastro autour de deux points :

- La réponse à l'une des principales questions posées en ouverture de la campagne est largement négative: il n'est pas possible d'identifier des vestiges importants des phases d'occupation Archaïque, Classique et Hellénistique dont proviendraient les nombreux blocs utilisés en remploi dans le kastro. En l'absence de données stratigraphiques, cette phase ne pourra être documentée que par une analyse plus fine de ces blocs eux-mêmes, et donc par une étude architecturale détaillée du kastro, qui fait défaut pour l'instant. Une telle étude architecturale, étendue à l'ensemble de la colline, permettrait également de mieux comprendre les rares vestiges préservés des fortifications hellénistiques.

- En revanche, nous avons pu montrer que Palaiokastro était un site important durant l'Helladique Ancien et Moyen, et que les niveaux de ces périodes sont bien préservés sur une partie de la colline. C'est là que réside le potentiel archéologique le plus prometteur du site. Les niveaux en place n'ont pu être observés que sur une petite fenêtre en PAL-5; il serait souhaitable, à l'avenir, de relancer les opérations avec des moyens humains et techniques plus importants, incluant la prospection géophysique de l'ensemble de la zone, et une fouille en aire ouverte sur une partie plus importante de la terrasse nord, pour mieux documenter cette occupation ancienne.

\section{Annexe 1 : inscription trouvée dans le secteur PAL-4 (Jules Buffet)}

Mégare, apothèque Benardi Meletiou, $\mathrm{n}^{\circ}$ PAX21/4025.1. Fragment de stèle en marbre blanc, brisée sur tous les côtés, découvert le $1^{\text {er }}$ septembre 2021 sur le secteur PAL-4, immédiatement au sud de la tombe TB1, dans un niveau de démolition. Dimensions : $h$. : $0,16 \mathrm{~m}, 1 .: 0,19 \mathrm{~m}$, ép. : 0,07 m. Hauteur des lettres : $1,8-2 \mathrm{~cm}$, interligne $0,8 \mathrm{~cm}$ (fig. 27).

Date : $\mathrm{II}^{\mathrm{e}}$ s. av. J.-C. - I ${ }^{\mathrm{er}}$ s. ap. J.-C.

Vacat

[- - - $] \Lambda$ EITO[- - - ]

$[---]$ K $\Lambda$ EIT[- - ] 


\section{[- - -]ẠIOșXạ[- - - ]}

Vacat

\section{Fig. 27. Inscription trouvée sur le secteur PAL-4.}

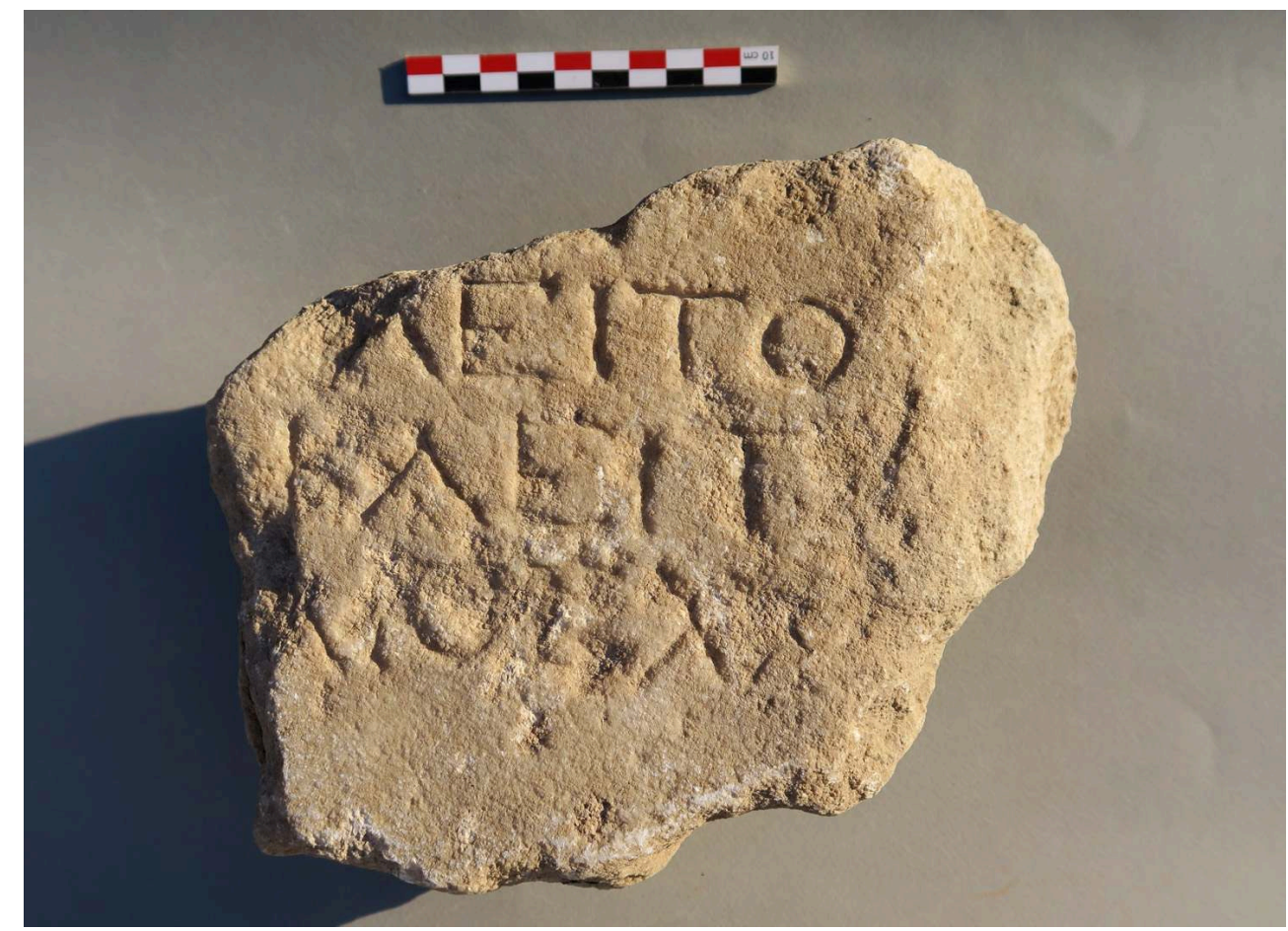

Cliché : L. de Barbarin

Bien que très fragmentaire, cette stèle est certainement un monument funéraire, car les deux premières lignes ne peuvent guère être que des noms propres. Le défunt portait un nom identique ou similaire à celui de son père, mais il est difficile de

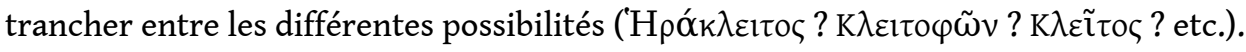




\section{BIBLIOGRAPHIE}

Mégare, Pachis, 2017

MÉGARE. - Pachis - 2017, Chronique des fouilles en ligne, n. 6889, mise en ligne le 23 juillet 2019, consultée le 25 octobre 2021. URL https://chronique.efa.gr/?kroute=report\&id=6889.

Mégare, Pachis, Aghios Georgios, 2018

MÉGARE. - Pachis, Aghios Georgios - 2018, Chronique des fouilles en ligne, n. 8508, mise en ligne le 15 juillet 2020, consultée le 25 octobre 2021. URL https://chronique.efa.gr/?

kroute=report\&id=8508.

ZORIDIS 1984

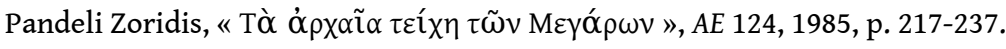

\section{NOTES}

1. Voir les notices publiées sur ces opérations : Mégare, Pachis, 2017 ; Mégare, Pachis, Aghios Georgios, 2018.

2. Dans le rapport préliminaire de la campagne de 2018, il est indiqué qu'il s'agit d'une statue masculine (Mégare, Pachis, Aghios Georgios, 2018). C'est là une erreur, due au fait que l'espace entre les pieds de la statue a été grossièrement creusé lors de son remploi (cf. infra), donnant faussement l'impression que les pieds étaient nettement séparés, comme sur une statue masculine. Sur les deux côtés de la statue, cependant, les plis abondants du chitôn qu'elle portait sous l'himation sont bien identifiables. Ce détail permet de restituer une statue féminine, dans un contrapposto comparable, par exemple, à celui de la Grande Herculanaise.

3. Thucydide, III, 51.

4. Voir sur ce point ZORIDIS 1984, p. 231-237.

5. Sur Agios Georgios, on compte plus de 21000 fragments correspondants à 2311 nombre minimum d'individus pondérés, sur Paleokastro, les premiers comptages indiquent environ 10000 fragments pour les sondages étudiés. 


\section{INDEX}

\section{Thèmes : EFA}

sujets https://ark.frantiq.fr/ark:/26678/pcrtaGFcSzXQ5x, https://ark.frantiq.fr/ark:/26678/ pcrtbptj4SOA1W, https://ark.frantiq.fr/ark:/26678/pcrtk6D8S79lNB

Année de l'opération : 2021

lieux https://ark.frantiq.fr/ark:/26678/pcrt8aDUwkuQGd chronologie https://ark.frantiq.fr/ark:/26678/pcrtZyHDNzd3Ie, https://ark.frantiq.fr/ark:/ 26678/pcrtucKPkJskZS, https://ark.frantiq.fr/ark:/26678/pcrtaFkrKZEXbs, https:// ark.frantiq.fr/ark:/26678/pcrtAQyKm9qosx

\section{AUTEURS}

\section{THIERRY LUCAS}

EFA

\section{PATRICK MARCHETTI}

Université catholique de Louvain

\section{PANAGIOTA AVGERINOU}

Éphorie des antiquités d'Attique occidentale

\section{LOU DE BARBARIN}

Aix-Marseille Université

JULES BUFFET

Université Paris-Nanterre

\section{ANTHONY PEETERS}

Université catholique de Louvain

LOUIS PIRAT

INRAP

BRIEUC GUILLAUME

EFA

MARILOU DE VALS

Sorbonne Université 